\title{
Detailed force modelling of the secondary load cycle
}

\section{Ghadirian, Amin; Bredmose, Henrik}

Published in:

Journal of Fluid Mechanics

Link to article, DOI:

$10.1017 / \mathrm{jfm} .2020 .70$

Publication date:

2020

Document Version

Peer reviewed version

Link back to DTU Orbit

Citation (APA):

Ghadirian, A., \& Bredmose, H. (2020). Detailed force modelling of the secondary load cycle. Journal of Fluid Mechanics, 889, [A21]. https://doi.org/10.1017/jfm.2020.70

\section{General rights}

Copyright and moral rights for the publications made accessible in the public portal are retained by the authors and/or other copyright owners and it is a condition of accessing publications that users recognise and abide by the legal requirements associated with these rights.

- Users may download and print one copy of any publication from the public portal for the purpose of private study or research.

- You may not further distribute the material or use it for any profit-making activity or commercial gain

- You may freely distribute the URL identifying the publication in the public portal

If you believe that this document breaches copyright please contact us providing details, and we will remove access to the work immediately and investigate your claim. 


\title{
Detailed force modelling of the secondary load cycle
}

\author{
Amin Ghadirian ${ }^{1} \dagger$, Henrik Bredmose ${ }^{1}$ \\ ${ }^{1}$ DTU Wind Energy, Nils Koppels Allé, Building 403, DK-2800 Kgs. Lyngby, Denmark
}

(Received xx; revised $\mathrm{xx}$; accepted $\mathrm{xx}$ )

Steep wave passage around vertical circular cylinders is associated with an additional force peak occurring after the main peak: the secondary load cycle. The secondary load cycle for a focused wave group typical of offshore wind turbine foundations at $33 \mathrm{~m}$ full scale water depth is investigated in scale 1:50. Ensemble averaged force, front face pressures and free surface elevation measurements are used as the basis for the investigation. A two-phase free-surface Reynolds Averaged Navier-Stokes (RANS) solver is validated against generic cases of turbulent flow over a wall, wave-boundary layer flow for Reynolds number, $R e$, of $1 \cdot 10^{4}<R e<1 \cdot 10^{7}, 2 \mathrm{D}$ drag on a cylinder for $1 \cdot 10^{2}<R e<$ $2.5 \cdot 10^{5}$ and $2 \mathrm{D}$ oscillatory flow for three combinations of $R e=\left\{5.8 \cdot 10^{4}, 9 \cdot 10^{4}, 1.7 \cdot 10^{5}\right\}$ and Keulegan-Carpenter number, $K C=\{6,12,18\}$, respectively. The solver is next applied to reproduce ensemble-averaged experimental results of the focused wave group and a good match for the inline force and free surface elevation is found along with a good match for the measured front face pressures.

The numerical solution for the focused wave is next analysed in detail to explain the cause of the secondary load cycle. We find that the secondary load cycle is confined to an upper region ranging from just above the still water level to 1.5 cylinder diameters below. By a further break down of the pressure field into contributions from the individual terms of the vertical Navier-Stokes equation, we find that the local force peak in the secondary load cycle is mainly caused by suction effects around the still water level on the backside, contributed through the material time derivative of the vertical velocity, $\frac{D \rho u_{z}}{D t}$. The suction occurs due to the rapid decrease of water level below the generated water column at the back of the cylinder, which at this time has only just begun its downward motion. The first force local minimum in the secondary load cycle is aided by the hydrostatic pressure from the water column while the second local minimum of the secondary load cycle is aided by wash-down effects on the front side.

Finally, the role of the observed vortices behind the cylinder is discussed and compared to reference computations with slip conditions. The results confirm findings from earlier slip boundary studies that the global force history through the secondary load cycle is not strongly affected by the boundary layer. The source of vortices behind the cylinder, observed in both sets of computations is discussed.

\section{Key words:}

$\dagger$ Email address for correspondence: amgh@dtu.dk 


\section{Introduction}

Nonlinear loads from steep waves are important for a safe design of offshore substructures. Higher-harmonic load components can induce ringing response and have received strong attention during the last three decades. Another phenomenon, related to force histories of highly nonlinear waves, is the secondary load cycle, which manifests itself by an additional peak in the inline force history close to the following force minimum. The secondary load cycle has sometimes been linked to ringing, see e.g. (Chaplin et al. 1997), although higher-harmonic forces are inherently present independently of whether a distinct secondary load cycle is present.

Several explanations have been suggested as the reason for the appearance of the secondary load cycle. However, there is not an agreement about the physical process which drives the secondary load cycle. The goal of the present paper is to study the cause of the secondary load cycle and the physical process behind it.

Grue et al. (1994) were the first to report the existence of what was initially called a secondary oscillation in the force-recordings. They characterized the starting time, period and amplitude of the oscillation in detail. They also reported that these oscillations occur about one-quarter of the wave period after the main peak in the loading and has a period of as long as $15 \%$ of the wave period, with $11 \%$ of the peak-to-peak amplitude of the total force. Grue et al. (1994) discussed that the experiments and the force recordings indicate the importance of the particle velocity under the wave crest and presence of the surface waves (which they related to the effect due to the gravity) in the problem. Hence, they suggested the Froude number, $F r=u / \sqrt{g D}$, as a defining non-dimensional parameter where $u$ is the particle velocity below the crest, $g$ is the gravitational acceleration and $D$ is the cylinder diameter. A criterion, $\mathrm{Fr}>0.4$, was suggested for the appearance of the secondary load cycle. They attributed the local phenomenon to a suction region one cylinder diameter below the still water level. The authors suggested that resonance between the free surface and the cylinder body may occur, which creates this suction and ultimately the secondary oscillation. Besides, the authors suggested that since the secondary oscillation happens one-quarter of the wave period after the main peak of the inline force (force in the mean wave propagation direction, $F_{x}$ ), the marine substructure might experience a build-up of resonant responses.

Chaplin et al. (1997) conducted a set of experiments for the response of a single vertical cylinder in the inertia regime in steep non-breaking waves including discussions on the secondary load cycle. They used spring-supports to adjust the stiffness of the cylinder, to place its natural frequencies in the range from 3 to 11 times the dominant wave frequency. The experiments were performed with three different cylinder diameters. It was found that the secondary load cycle has a significant effect on the response of the structures with natural frequencies close to the frequency of the secondary load cycle. The magnitude and period of the cycle were comparable to the results from Grue et al. (1994), with a magnitude between $8 \%-12 \%$ of the total force range for the smallest and largest diameter cylinder respectively and a period of $15 \%$ of the main force cycle period. They suggested that the secondary load cycle only appears significantly when $\mathrm{Fr}>0.6$. They further suggested that $F r$ is neither the sole influential parameter on the magnitude of the secondary load cycle nor the most important one. Instead, they proposed that the wave steepness is more influential in the magnitude of the secondary load cycle and for the largest waves the secondary load cycle magnitude decreases with increasing steepness while it is proportional to the cylinder diameter. They suggested that there is a direct relationship between the cubed diameter and the magnitude of the secondary load cycle. 
Further, the importance of the secondary load cycle in the ringing of the response cylinder was discussed.

Later on, Grue (2002) extended the analysis by more measurements and while he reported the same approximations for the relative magnitude and period of the secondary load cycle as in the initial investigation (Grue et al. 1994), he mentioned that appearance of the secondary load cycle depends on both cylinder diameter and the wave amplitude relative to the wave length. He reported that the secondary load cycle occurs when the wave length is larger than approximately 10 times the cylinder diameter. Grue (2002) stated that the secondary load cycle does not happen in the lab when the scale is too small and explained this phenomenon by the presence of flow separation. Grue \& Huseby (2002) investigated in more detail the effects of the scale factor. They concluded that in the small scale, the secondary load cycle happens when the wave slope, $k \eta_{m}$, exceeds 0.3 and for wave length ratio, $k R$, smaller than 0.33 where $k$ is the wave number, $\eta_{m}$ is the crest height and $R$ is the cylinder radius. In the moderate scale, the secondary load cycle is more visible for waves with smaller slopes. The difference between the experiments in small and moderate scale was explained by the effect of flow separation. According to this work the secondary load cycle gives an important contribution to build-up of resonant body responses for a natural frequency of the structure about four times the local wave frequency.

Rainey (2007) associated the secondary load cycle with the violent motion of the water surface. A cavity bubble is formed behind the cylinder which then collapses to give the secondary load cycle. He reported no connection between the secondary load cycle and the third harmonic of the wave frequency.

Paulsen et al. (2014b) investigated the secondary load cycle by computation of the interaction of regular stream function theory waves and a vertical circular cylinder. They concluded that for the analyzed cases, the secondary load cycle was associated with force components at frequencies above the fifth and sixth harmonic of the fundamental wave frequency. It was also found that the magnitude of the secondary load cycle increases for decreasing values of $k R$ in agreement with observations of Grue (2002). The magnitude and period of the secondary load cycle were shown to depend largely on $H / H_{\max }$ and to a lesser extent to $k h$ for the analyzed cases where $H$ is the wave height, $H_{\max }$ is the limiting wave height for a regular wave and $h$ is the water depth. They also stated by visual observation and a simplified analytical model that the secondary load cycle was caused by the free surface which drives a return flow from the back of the cylinder after the passage of the wave crest. A distinct vortex pair at the downstream side of the cylinder was shown to appear during the presence of a secondary load cycle. A simple potential flow model was used, to deduce that the force contribution from the secondary load cycle may be caused by the upstream propagating flow towards the front side of the cylinder and the associated downstream vortex pair.

In line with Rainey (2007), Jose et al. (2017) explained the creation of the secondary load cycle by the blockage of the flow by the cylinder being filled by the diffracted waves, when they meet at the down stream side, and the resulting hump of water piled up at the back of the cylinder. This hump of water would create high pressure which would act in the opposite direction to that of the flow and exert a negative force on the back of the cylinder. Jose et al. (2017) also stated that the correct turbulence modelling would contribute to an accurate estimation of the secondary load cycle. They did not, however, explain the reason for the importance of the turbulence in the characteristics of the secondary load cycle.

Kristiansen \& Faltinsen (2017) suggested that the flow separation (governed by KC) results in the local rear run-up. They conclude that the local rear run-up leads to the 
secondary load cycle, since it gives rise to the fourth and the fifth harmonics while reducing the third harmonic

Riise et al. (2018) investigated 2166 individual waves and separated the harmonics larger than $3.5 \omega_{T T}$ from the force time series, where $\omega_{T T}$ is the trough to trough angular frequency of the wave. Regression methods were used to investigate the effect of $K C$, governing flow separation and $F r$, free surface gravity wave effects, on the magnitude of the secondary load cycle. They concluded that both parameters are important while the best correlation was found between the secondary load cycle magnitude and an improved version of $\mathrm{Fr}$. This may be explained through the initial definitions of $\mathrm{Fr}$ and $K C$ in this study, which leads to $F r=K C \sqrt{k D} / 2 \pi$ at deep water, such that $F r$ offers an alternative, but not independent, ordering of the conventional properties $K C$ and $k D$ in the full parameter space. They showed a limiting threshold of $K C \approx 4-5$ or $F r \approx 0.3-0.4$ which indicates a change in the physical mechanisms that govern the high-frequency force.

Other authors (Ghadirian et al. 2016, 2017) have reported the presence of the secondary load cycles in lab measurements and computations without explaining the source of the phenomenon. From the literature review, it is observed that although the secondary load cycle is clearly a result of the wave-structure interaction and often linked to the flow at the backside of the cylinder, no precise account of the detailed flow mechanics causing it has been provided. The main goal of the present paper is to investigate the physical process in which the secondary load cycle is created. Experimental and numerical results of a focused wave group in one sea state are extensively used and analysed to separate the flow in different terms and identify the source of the secondary load cycle.

In section 2 the experimental setup and the model are described. The models used and the numerical schemes with modified turbulence models implemented in OpenFOAM are defined. To increase the trust in the model, an extensive validation study for a set of basic cases is performed and presented in section 3. The cases include single-phase steady and oscillating flow around a cylinder which are close to the flow regimes of the investigated focused wave group. Afterwards, in section 4 the numerical results are validated in terms of free surface elevation, inline force and local pressure against measurements. Section 5 includes a detailed investigation of the flow by dividing the flow into horizontal disks and by calculating the pressure contribution from separate terms of the vertical NavierStokes equation. The role of the structural boundary layer and the force contribution from the vortices at the back of the cylinder is investigated in section 6 by comparison to a force computation with slip boundary conditions on the cylinder. Concluding remarks and suggested future work are presented in the last section 7 .

\section{Methodology}

\subsection{Experiments}

The experiments were conducted at DHI Denmark in scale 1:50 as part of the DeRisk project (Bredmose et al. 2016). The used shallow water wave basin was $35 \mathrm{~m}$ long and 25 $\mathrm{m}$ wide. The investigated focused wave group was generated using a JONSWAP spectrum with laboratory-scale significant wave height of $0.15 \mathrm{~m}$ and peak period of $2.12 \mathrm{~s}$ at a water depth of $0.66 \mathrm{~m}$. These conditions correspond to a 10 year return period climate in typical North Sea conditions close to the German Bight. The chosen wave height for the focused wave group (Tromans et al. 1991) was 1.86 times the significant wave height, $H_{s}$, of this sea state with no inclusion of directional spreading. Although the whole wave group was generated in the laboratory and numerically, only the results of the main wave 
in the focused wave group are shown in the present paper. The monopile was equipped with two force transducers, one at the top and one at the bottom of the cylinder with a laboratory scale diameter of $0.14 \mathrm{~m}$. Based on the peak frequency wave number, these conditions are equivalent to $k h=0.85$ and $k R=0.09$.

Five pressure sensors were installed on the front side of the cylinder facing the wavemakers. The test was repeated 8 times and the used measurements are ensemble-averaged over the 8 repetitions. Linear wave generation theory (Dean \& Dalrymple 1991) was used to calculate the piston type wave-maker position signals in the lab.

Reflection analysis was performed on the measured free surface elevation based on the work of Goda \& Suzuki (1976) and Bredmose et al. (2010) and less than $5 \%$ reflection was observed from the artificial beach in the studied test.

Several methods were used to estimate $K C$ and $R e$ values in the focused wave group defined as

$$
\begin{aligned}
& K C=\frac{U_{m}}{f_{p} D} \\
& R e=\frac{U_{m} D}{\nu} .
\end{aligned}
$$

Here $U_{m}$ is either the maximum or the standard deviation of the horizontal velocity of the flow, $D$ is the diameter of the cylinder, $f_{p}$ is the peak frequency of the wave spectrum and $\nu$ is the kinematic viscosity of the fluid. The calculated non-dimensional numbers depend on choosing maximum or standard deviation of the velocity and the depth at which the velocity is sampled. In the experiments, the velocity was not measured. However, using the fully nonlinear potential flow solver OceanWave3D (Engsig-Karup et al. 2009), the velocity time series at any height was calculated. Following Sumer \& Fredsøe (2006), $K C$ and $R e$ were calculated using the standard deviation of the velocity at the still water level and were equal to 6 and $1 \cdot 10^{4}$ respectively. Choosing the maximum velocity value at the maximum crest height (Yang \& Rockwell 2002) leads to a KC value of 18 and a $R e$ value of $1.7 \cdot 10^{5}$ and at the half depth (Yang \& Rockwell 2002) leads to $\mathrm{KC}$ and $R e$ values of 6.4 and $5.8 \cdot 10^{4}$ respectively. The calculation of the $K C$ and $R e$ is important for recognition of the flow regime around the cylinder.

\subsection{The numerical model}

The coupled solver OceanWave3D-Waves2Foam (Paulsen 2013; Paulsen et al. 2014a) was used to reproduce the experiment. Waves2Foam is an extension to the InterFoam solver of OpenFOAM (Weller et al. 1998) which uses relaxation zones to induce incident gravity waves (Jacobsen et al. 2012) in the domain. InterFoam uses a volume of fluid (VOF) method to treat the free surface flow (Hirt \& Nichols 1981). It solves the continuity equation and the momentum conservation equations,

$$
\begin{gathered}
\frac{\partial u_{i}}{\partial x_{i}}=0 \\
\frac{\partial \rho u_{i}}{\partial t}+u_{j} \frac{\partial \rho u_{i}}{\partial x_{j}}=-\frac{\partial p^{*}}{\partial x_{i}}-g_{j} x_{j} \frac{\partial \rho}{\partial x_{i}}+\frac{\partial}{\partial x_{j}}\left(2 \mu_{\mathrm{eff}} S_{i j}\right)+\sigma_{T} \kappa \frac{\partial \alpha}{\partial x_{i}} \\
S_{i j}=\frac{1}{2}\left(\frac{\partial u_{i}}{\partial x_{j}}+\frac{\partial u_{j}}{\partial x_{i}}\right)
\end{gathered}
$$




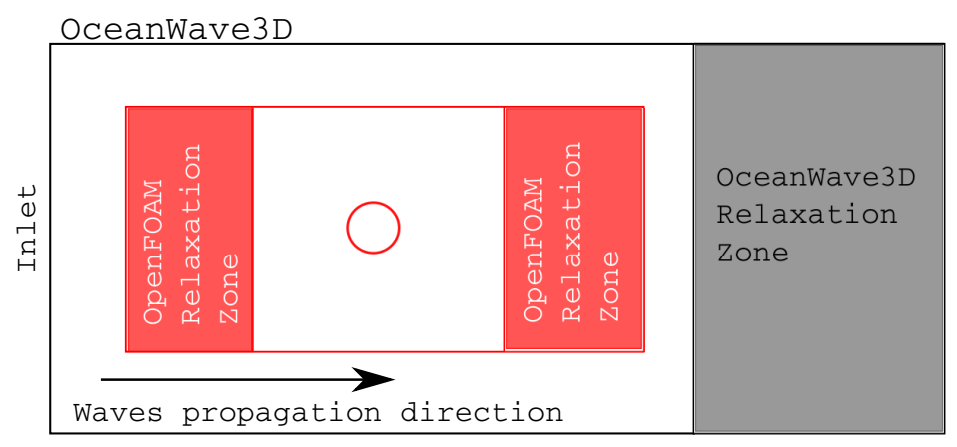

Figure 1: Schematic drawing of the numerical domains including the potential flow fully nonlinear solver OceanWave3D domain and the Navier-Stokes solver OpenFOAM domain.

where $u_{i}$ are the velocity components, $x_{i}$ are the coordinates, $\rho$ is the local fluid density, $p^{*}$ is the pressure minus the hydrostatic potential $\rho g_{j} x_{j}, g_{j}$ is the gravitational acceleration, $\mu_{\text {eff }}$ is the local effective viscosity and $S_{i j}$ is the mean strain rate tensor. The effective viscosity, $\mu_{\mathrm{eff}}$, is calculated as $\mu_{\mathrm{eff}}=\mu+\mu_{t}$, where $\mu$ is the local dynamic viscosity and $\mu_{t}$ is the turbulent viscosity (Menter et al. 2003). The turbulent viscosity was set to zero for the computations with a slip boundary condition on the cylinder wall. The viscosity of the fluid, however, was considered in all of the computations. The fluid fraction is denoted by $\alpha$ which can take values between 0 and 1 for full air and water occupation of the cells respectively. Local fluid properties are calculated by linear weighting between the water and air properties, e.g. $\Phi=\alpha \Phi_{\text {water }}+(1-\alpha) \Phi_{\text {air }}$, where $\Phi$ represents any needed fluid property. Hence, $\mu_{\text {eff }}=\alpha \mu_{\text {water }}+(1-\alpha) \mu_{\text {air }}+\mu_{t}$ in each cell. The last term in (2.4) takes the surface tension, $\sigma_{T}$, into account by considering the surface curvature, $\kappa$, in the border of the two phases where the gradient of $\alpha$ is non-zero. In our calculations, this term is dismissed.

The transport equation of $\alpha$ is

$$
\frac{\partial \alpha}{\partial t}+\frac{\partial \alpha u_{j}}{\partial x_{j}}+\frac{\partial}{\partial x_{j}}\left(\alpha(1-\alpha) u_{j}^{r}\right)=0
$$

where $u_{j}^{r}$ has the unit of velocity and is in the normal direction to the air-water interface. The last term compresses the region where $\alpha$ is between 0 and 1 . More information on the performance of this term can be found in Deshpande et al. (2012).

To reproduce the measurements, the wave paddle signal was created from the first-order wave generation similarly to the experiments (Dean \& Dalrymple 1991) and the velocity of the paddles was used as a flux boundary condition in the non-linear potential flow solver OceanWave3D (Engsig-Karup et al. 2009). This method of generation is linearly consistent with the piston wave generation in the lab. Differences at higher orders are expected because no piston or boundary movement is included in the model. However, good consistency with experiments using this approach has been presented in earlier work Paulsen et al. (2014a) and as part of DeRisk project Bredmose et al. (2016) and Ghadirian et al. $(2016,2017)$. A top view of the computational domains is shown in figure 1 . The embedded OpenFOAM domain was driven with waves generated in OceanWave3D through a relaxation zone (Jacobsen et al. 2012).

Convergence tests were performed to verify that all the significant waves in the sea state, even with the highest frequency were resolved. From these studies, it was observed that in the OceanWave3D domain a discretization by $501 \times 1 \times 15$ cells with constant time step $\delta t=0.01 s$ was numerically converged more than $99 \%$ meaning that the results 
would change less than $1 \%$ by refining the mesh. The OceanWave3D results were mapped inside the OpenFOAM domain in each time step in a 3 meters long relaxations zone. The OpenFOAM mesh with $\mathrm{d} x=0.02 \mathrm{~m}$ and with a cell aspect ratios close to one showed less than $1 \%$ change in the free surface elevation and inline force results when the mesh resolution was doubled in all directions. Nevertheless, for better resolution of the vortices around the cylinder, the finest mesh was used with $\mathrm{d} x=0.01 \mathrm{~m}$ and 24 million cells.

For turbulence modelling, the $\mathrm{k} \omega$-SST model implemented in OpenFOAM was used. The implementation is based on Menter et al. (2003) without the density in the transport equations in OpenFOAM which was added following the work of Brown et al. (2014) and Devolder et al. (2017). The resulting equations are

$$
\begin{aligned}
& \frac{\partial \rho k}{\partial t}+\frac{\partial \rho u_{j} k}{\partial x_{j}}-\frac{\partial}{\partial x_{j}}\left(\Gamma_{k} \frac{\partial k}{\partial x_{j}}\right)=\rho P_{k}-\beta^{*} \rho \omega k \\
& \frac{\partial \rho \omega}{\partial t}+\frac{\partial \rho u_{j} \omega}{\partial x_{j}}-\frac{\partial}{\partial x_{j}}\left(\Gamma_{\omega} \frac{\partial \omega}{\partial x_{j}}\right)=\rho \frac{\gamma}{\nu_{t}} G-\beta \rho \omega^{2}+\rho 2\left(1-F_{1}\right) \frac{\sigma_{\omega 2}}{\omega} \frac{\partial k}{\partial x_{j}} \frac{\partial \omega}{\partial x_{j}}
\end{aligned}
$$

where

$$
\begin{array}{r}
\Gamma_{k}=\mu+\mu_{t} \sigma_{k}, \Gamma_{\omega}=\mu+\mu_{t} \sigma_{\omega} \\
G=\nu_{t} \tau_{i j} \frac{\partial u_{i}}{\partial x_{j}}, P_{k}=\min \left(G ; c_{1} \beta^{*} k \omega\right), \\
\mu_{t}=\rho \frac{a_{1} k}{\max \left(a_{1} \omega ; S \cdot F_{2}\right)} .
\end{array}
$$

The constants, $\sigma_{k}, \sigma_{\omega}, \beta$ and $\gamma$ are chosen and computed according to Brown et al. (2014) among others. The resulting $k$ and $\omega$ from solving (2.7) and (2.8) are used to calculate $\mu_{t}$ required in $(2.4)$.

The convergence tests were performed to find the optimum combination of Courant number and the discretization schemes based on the work by Larsen et al. (2018). A summary of the model setup is presented in table 2 in Appendix A. The upwind scheme was used for the divergence term of the turbulence quantities $k$ and $\omega$.

The coupling of the pressure and velocity equations was done by the PIMPLE solver. PIMPLE is a combination of two algorithms Semi-Implicit-Method-Of-PressureLinked-Equations (SIMPLE) and Pressure-Implicit-Split-Operator (PISO). While "smoothsolver" was used for $\alpha, k$, and $\omega$ linear equations, the pressure equation was solved by the generalised Geometric-Algebraic Multi-Grid (GAMG) and the velocity by the Preconditioned Bi-Conjugate Gradient (PBiCG) with Diagonal Incomplete-LU (DILU) pre-conditioner (Greenshields 2015). It was observed that the most consistent results to the measured free surface elevation were obtained when the number of outer corrections in the PIMPLE solver was set to one, which practically makes this solver the PISO solver. It was checked that the residuals were below $10^{-3}$ in all the computations to make sure the results are converged in each time step.

The boundary conditions of the domain are shown in table 3 in Appendix A. The boundary conditions on the sea bed and the side walls were of type zeroGradient for all quantities. The boundary condition of the velocity on the cylinder wall was defined as fixed zero in this table. However, computations with slip boundary condition on the cylinder were also performed to compare its effect. The length of the domain was defined by the wave length and the fact that the relaxation zones in the OpenFOAM domain should be at least as long as the longest wave mapped in the domain (Jacobsen et al. 2012; Jacobsen 2017). However, since the focused wave group contains very long waves in the spectrum of the sea state a reflection analysis was performed to find the shortest 
relaxation zone length for which reflected waves are smaller than $5 \%$. In addition, it was observed that the quality of propagation of the waves depends drastically on the aspect ratio of the cells around the free surface. The aspect ratio should be as close to one as possible and even an aspect ratio of 2 diminishes the performance of the solver (Jacobsen 2011; Paulsen 2013; Larsen et al. 2018). This limitation in the aspect ratio limited the ability of resolving the boundary layer at the cylinder wall. It was also observed that refining the mesh in the radial direction around the cylinder introduced artificial waves around the cylinder which affects the inline force time series on the cylinder. Hence using a uniform mesh in the domain with a wall function on the cylinder wall was found to be the most viable solution.

\section{Model validation against basic flows}

The numerical setup with the same discretization as described in the previous section was validated against cases of channel flow and steady and oscillating single-phase flow around a cylinder.

The simplest validation case was to investigate if the model is capable of capturing the law of the wall of a steady channel flow over a smooth wall. In plot (a) of figure 2, the validity of the model is shown in comparison to theory from von Karman (1931).

The theoretical equations used in this plot are given by

$$
\frac{\bar{u}}{U_{f}}=\left\{\begin{array}{rr}
y^{+} & y^{+}<5 \\
2.5 \ln y^{+}+5 & 30<y^{+}<500
\end{array} .\right.
$$

Here $y^{+}=\frac{y U_{f}}{\nu}$ is the non-dimensional wall distance, $U_{f}$ is the friction velocity, $\nu$ is the kinematic viscosity, $\bar{u}$ is the average velocity and $y$ is the normal distance from the wall. The viscous sublayer, buffer layer and log-law region are resolved and predicted very well in the inner layer of the flow.

The validity of the model in comparison with the measurements conducted by Jensen et al. (1989) in the calculation of the friction coefficient in the wave boundary layer over a smooth wall is shown in the plot (b). Two sets of computations were performed to validate the model with and without using the wall function respectively. The first set of the computations, carried out for a range of $R e$ values from $8.5 \cdot 10^{3}$ to $1.6 \cdot 10^{6}$, resolved the boundary layer with $y^{+}$values smaller than 1 in the first cell for all cases. The results of this group are consistent with the measurements except in the transition region, $1.5 \cdot 10^{5}<R e<7 \cdot 10^{5}$. It should be noted that the behaviour of the flow in this region is in general associated with some scatter for the friction coefficient since different experiments also obtain different friction coefficients (Jensen et al. 1989; Fredsøe et al. 2003). The second group of simulations was for a larger range of $R e$ values with $y^{+}$values larger than 30 next to the wall. The results for this group for $R e$ values larger than $1 \cdot 10^{5}$ are also consistent with the experiments.

To validate the model in flows around bluff bodies, cases of steady flow around a cylinder with different $R e$ values were simulated and the drag coefficient $\left(C_{d}\right)$ and Strouhal number $(S t)$ were compared with experimental results represented in Sumer \& Fredsøe (2006) and computational results from Rosetti et al. (2012), Stringer et al. (2014) and Ye \& Wan (2017). Here $S t=f D / u$ where $f$ is the vortex shedding frequency, $D$ is the cylinder diameter and $u$ is the flow velocity. It is shown in figure 3 that the results obtained using the current model and setup can give among the most consistent results with the measurements up to $R e$ of $1 \cdot 10^{5}$. However, the results for $R e=1 \cdot 10^{6}$, in the super critical region, are not as consistent with the measurements. The inconsistency 
(a)

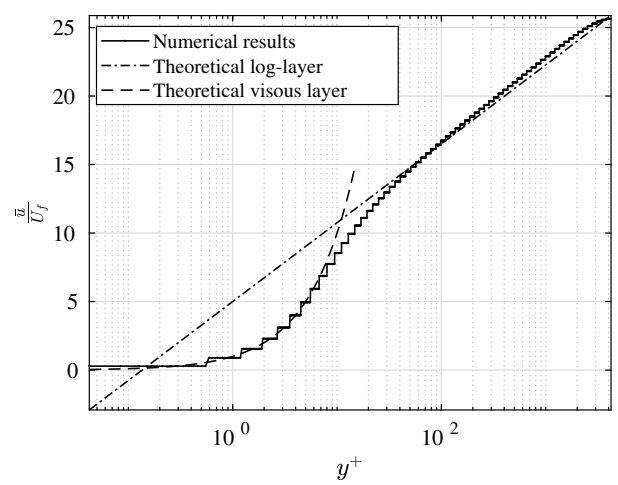

(b)

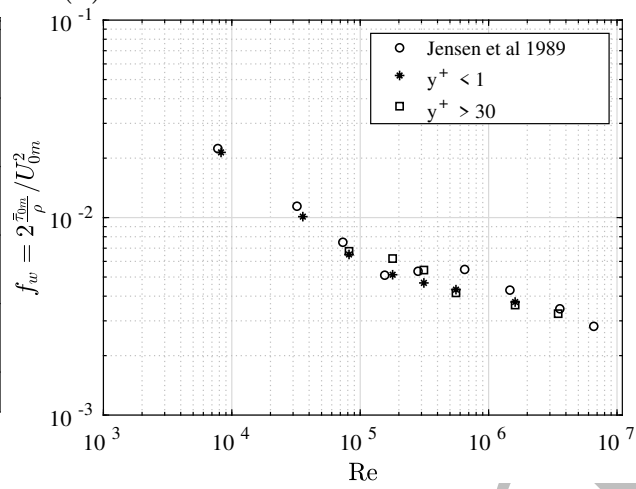

Figure 2: (a) The law of the wall. The solid black line shows the model results while the dashed lines show the theory from (3.1) (b) Wave boundary layer.

in this range is expected since the RANS simulations do not usually capture the correct behaviour of the flow above the subcritical regime (Rosetti et al. 2012; Stringer et al. 2014; Ye \& Wan 2017). For the cases of the present paper, Re is below $1.7 \cdot 10^{5}$. The solver is therefore considered sufficiently accurate for the flow regimes studied.

The next validation case is $2 \mathrm{D}$ oscillatory flow around a circular cylinder. In figure 4 the drag and inertia coefficients for three Reynolds and $K C$ values corresponding to the free surface flow at the crest, still water level and in half depth of $0.33 \mathrm{~m}$ are shown. In these computations the wall function was used on the cylinder wall and the boundary layer was not resolved. From plot (a) in this figure it is observed that the inertia coefficient is over predicted less than $3 \%$ in all cases with $K C$ values of 6,12 and 18 . For the drag coefficient there is an under prediction of less than $14 \%$ as shown in the plot (b) of figure 4 . In general a good agreement between the simulations results and the measurements is shown.

\section{Validation of the model results with experiments}

We now turn to the re-computation of the ensemble-averaged focused wave group. In figure 5 the ensemble-averaged measured time series of inline force is presented in the top plot. The standard deviation between the eight repetitions of the inline force and free surface elevations is shown with the grey area around the mean value curves. However, because of high repeatability, the deviation areas are hardly visible in this plot. It is observed that the maximum inline force happens at $14.8 \mathrm{~s}$ hereunder referred to as the first peak. The horizontal axis of the plots is limited to the event of interest, the secondary load cycle. Six time instances from $15 \mathrm{~s}$ to $15.5 \mathrm{~s}$ every $0.1 \mathrm{~s}$ are also shown in this plot with dotted lines for clarity. Two local minima are observed in the force time series at $15.1 \mathrm{~s}$ and $15.32 \mathrm{~s}$ with a maximum in between which hereunder are referred to as the first dip, the second dip and the second peak. The simulation results of inline force are presented in the same plot. The OpenFOAM results are in good agreement with the measurements. OpenFOAM also shows a good reproduction of the secondary load cycle phenomenon.

The free surface elevation at the monopile center (measured 5 diameters away from the cylinder) is shown in the middle plot. The measured time series is maximum at $14.92 \mathrm{~s}$ which indicates a phase shift between the inline force and the free surface elevation which is in agreement with the known characteristics of an inertia-dominant flow. The 
(a)

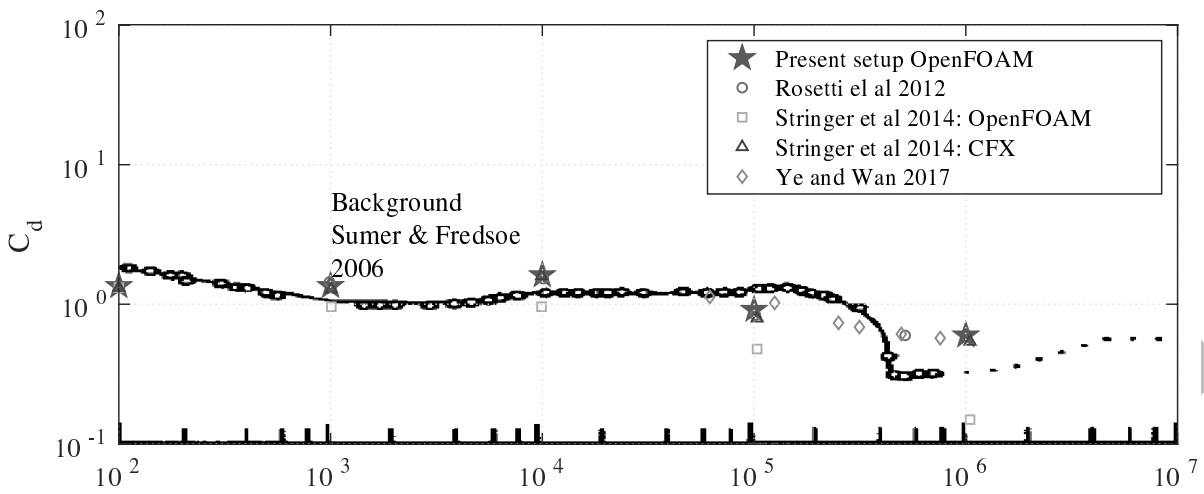

$\operatorname{Re}$

(b)

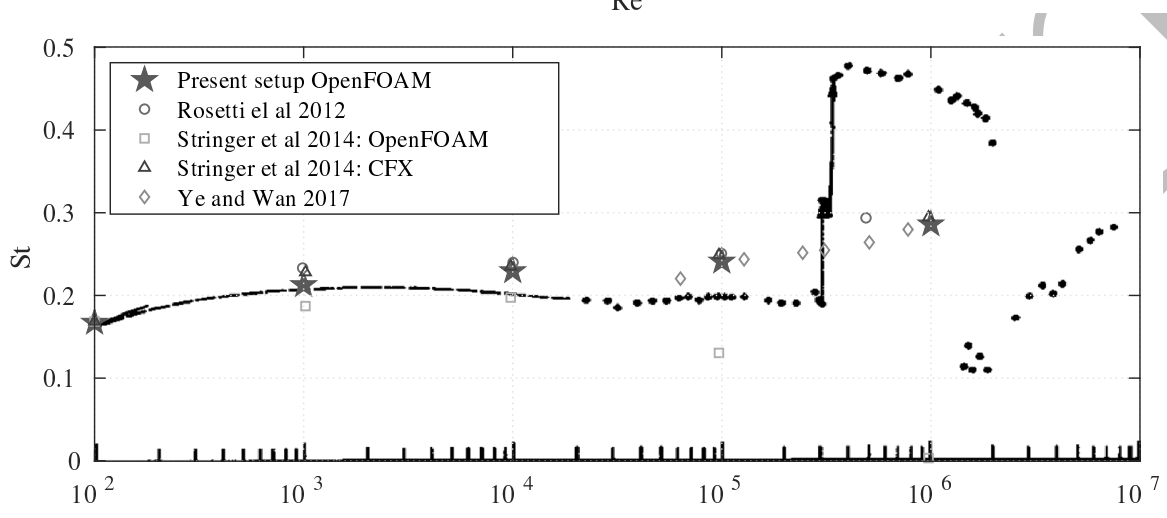

Figure 3: (a) Drag coefficient as a function of Re for a steady flow around cylinder. (b) $S t$ as a function of Re for a steady flow around cylinder. Results from three studies (Rosetti et al. 2012; Stringer et al. 2014; Ye \& Wan 2017) are included for comparison. The background plot shows the experiments represented in Sumer \& Fredsøe (2006).
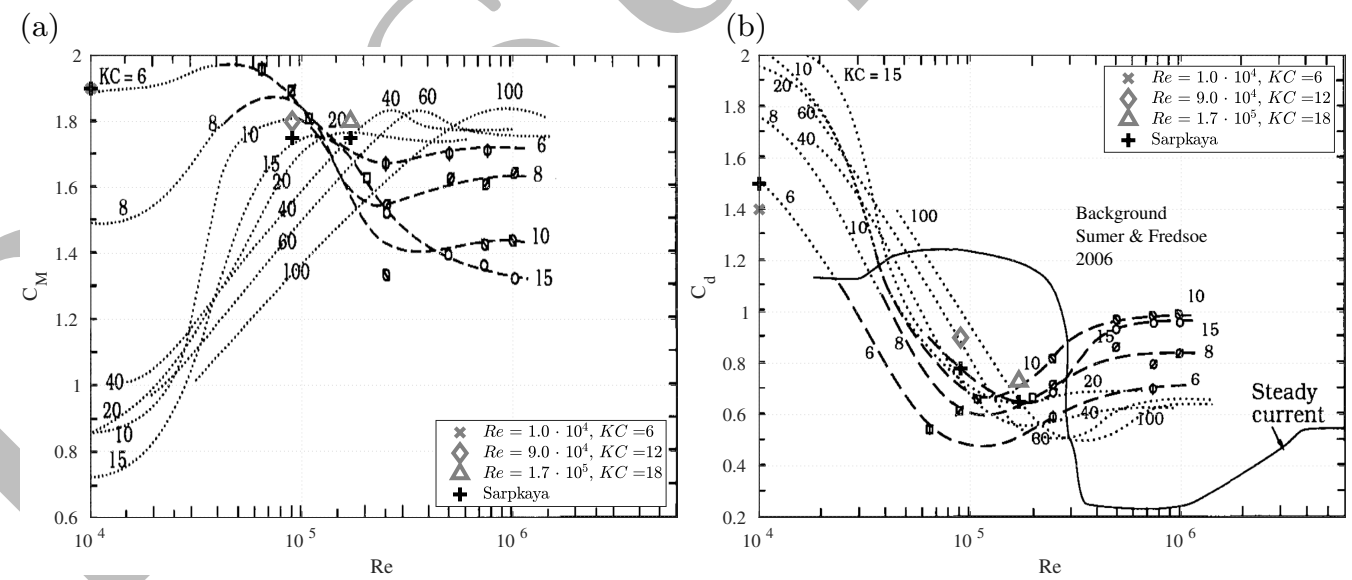

Figure 4: Drag and inertia coefficients of oscillating flow around the cylinder with three Reynolds and $K C$ covering the flow regimes of the free surface flow at three heights. (a) Inertia Coefficient. (b) Drag Coefficient. The background plot shows the experiments represented in Sumer \& Freds $\varnothing \mathrm{e}$ (2006). 

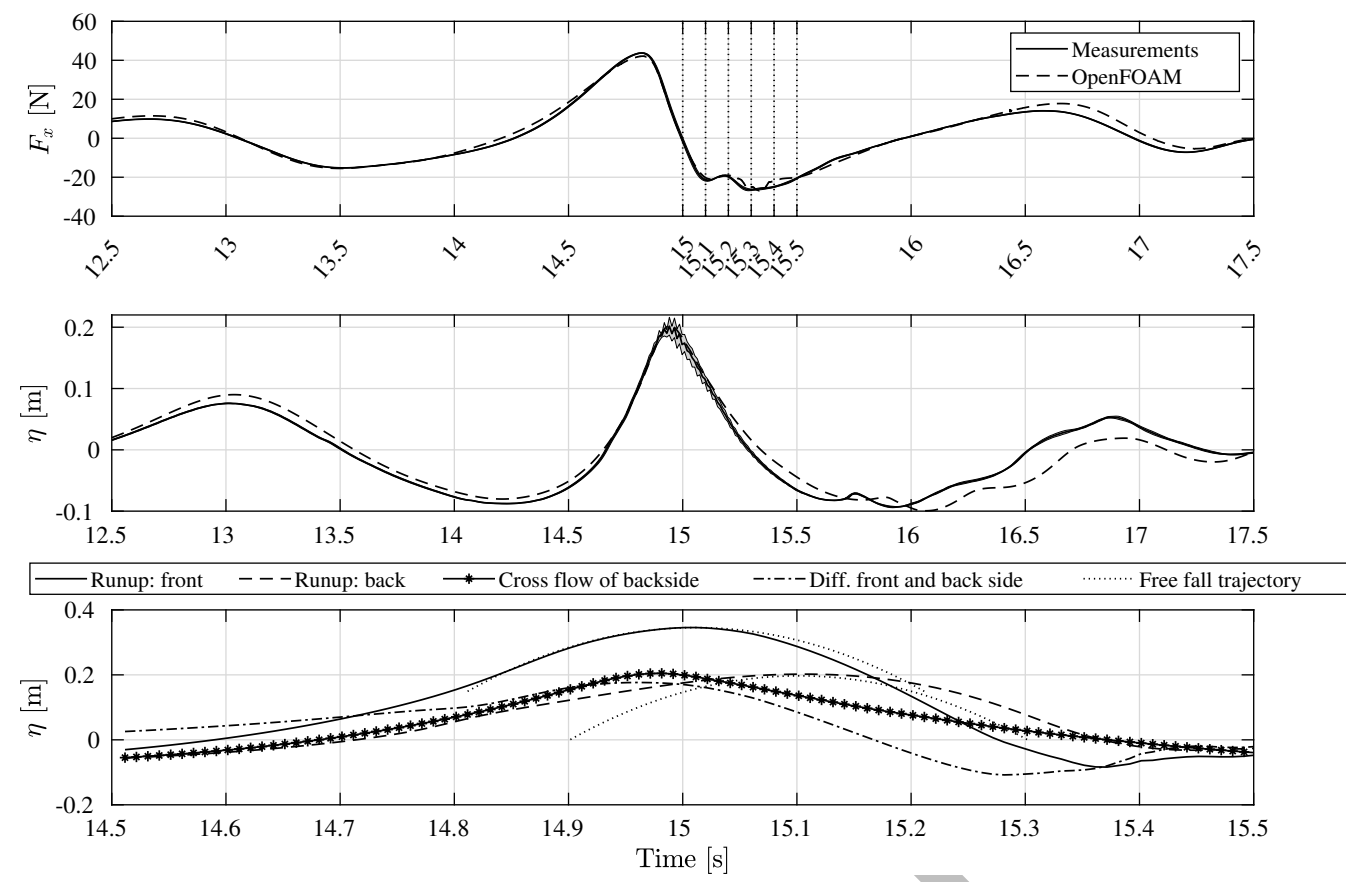

Figure 5: Top: Inline force time series. Middle: Free surface elevation. Bottom: Run-up from the OpenFOAM simulations.

free surface elevation is also presented in this plot and shows a good agreement with the measurements. However, the measured wave is slightly more symmetric than the computations. Since the wave paddles in the computations were only linearly consistent with the experiments these minor differences are expected.

In the bottom plot the run-up on the front and back side of the cylinder and the difference between them is shown from the OpenFOAM results. In addition, a free shoot and fall trajectory from the maximum run-up on both sides is presented. The water on the front side is thrown up at $14.85 \mathrm{~s}$ with only gravity imposing a force on it. Hence its trajectory is similar to the free shoot trajectory. After $15 \mathrm{~s}$ the run-up water falls faster than free fall. On the back side the run-up water is moved up and down slower than free shoot and fall peaking at $15.1 \mathrm{~s}$. The difference in the run-up on the front and the back side is a simple representation of the possible hydrostatic force contributions to the force history from the front and back flows. From the curve though a different time scale than the secondary load cycle is visible. The secondary load cycle can thus not be explained by hydrostatic effects related to the run-up and down flows at the front and back side.

The wave induced pressure time series measured at 5 heights of $0.16 \mathrm{~m}, 0.12 \mathrm{~m}, 0.08 \mathrm{~m}$, $-0.14 \mathrm{~m}$ and $-0.18 \mathrm{~m}$ from the still water level in the experiments are shown in figure 6 . The measurement height is presented in the title of each plot. The wave induced pressure is calculated as

$$
p_{\text {wave }}=\left\{\begin{aligned}
p_{\text {total }}+\rho g z, & z<0 \\
p_{\text {total }} & z \geqslant 0
\end{aligned}\right.
$$

which represents the pressure added to the gauge pressure in calm water conditions.

From figure 6 we can see that the pressure sensors that are always wet show positive and negative wave induced pressure similar to a sine wave, but the pressure sensors that 

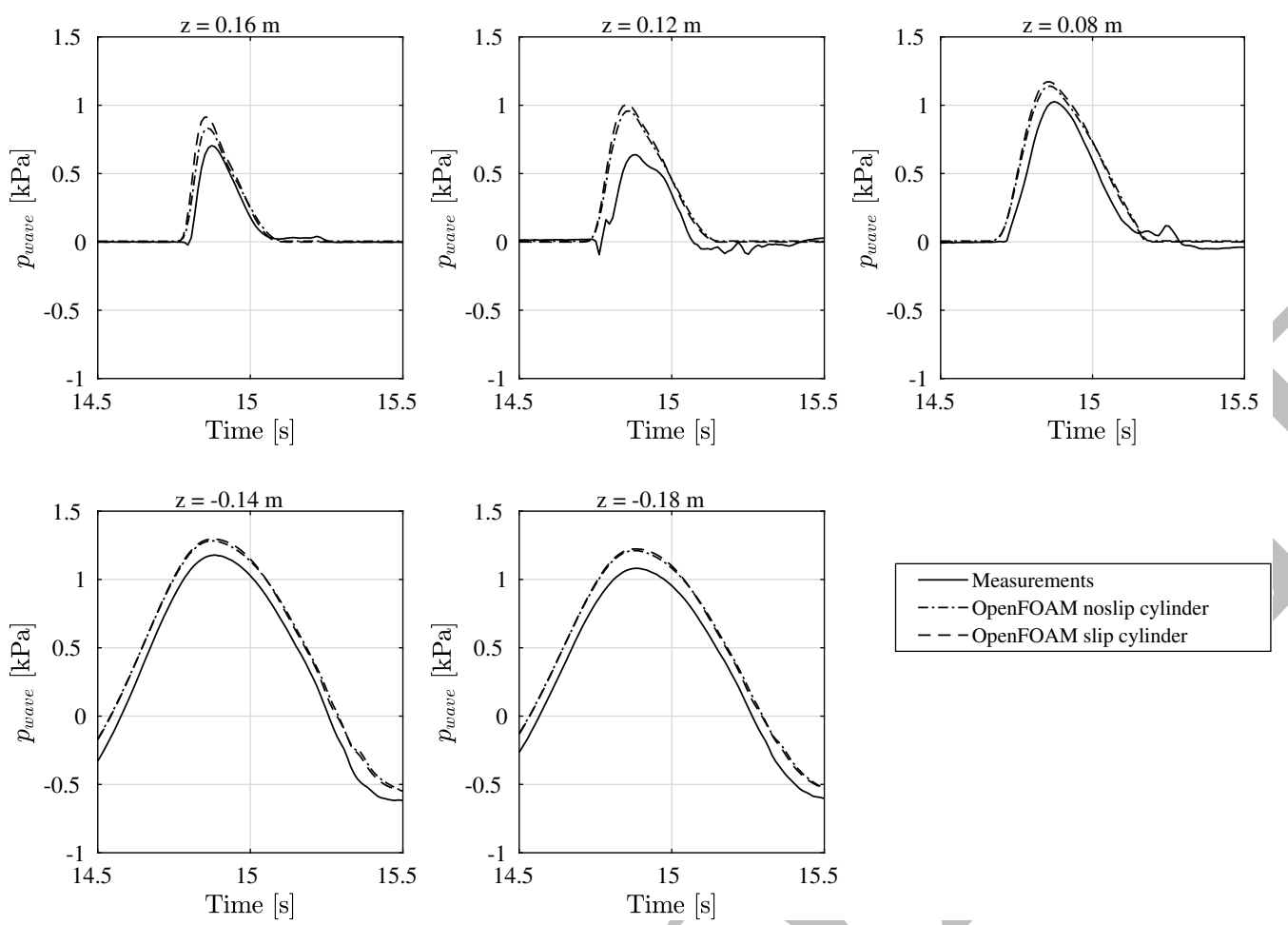

Figure 6: Wave induced pressure at five different heights on the front side of the cylinder.

are above the still water level and are sometimes dry show nonzero values only when wet during the run-up time on the front side. It is worth mentioning that the maximum wave induced pressure measured at $12 \mathrm{~cm}$ above the still water level is smaller than the ones measured at higher and lower heights. Small wriggles in the pressures above still water level are seen right after they become dry. This is likely to be a spurious feature of the pressure sensor or due to aeration effects.

In the same figure, wave induced pressures from computations with slip and noslip boundary condition on the cylinder are shown. The differences between the two calculations are very small and the agreement between the results and the measurements is generally good. The main differences are a small offset for the lower transducers, some amplitude difference for the upper transducers and lower maximum pressure at $12 \mathrm{~cm}$ than at $16 \mathrm{~cm}$. These are very likely related to slight differences in the waves that are reproduced in the computations. The absence of surface tension in the numerical setup may also have an effect on the run-up height and the resulting pressures.

Even with these observed differences, we note that the temporal variation of the pressures is very well reproduced. Given further the good match of the inline force and free surface elevation, we regard the numerical results to be a sufficiently accurate reproduction of the physical wave-structure interaction to warrant further detailed analysis of the secondary load cycle and the associated flow. 


\section{Detailed investigations}

In figure 7 the free surface of the flow is shown for the 6 time instants marked with dotted lines in the top plot of figure 5 . In plot (a), at $15.0 \mathrm{~s}$, the run-up on the front side is maximum. The wave crest is just on the back side of the cylinder. A distinct water column (mound) has already been formed at the back side. In plot (b), at $15.1 \mathrm{~s}$, the run-up on the back side starts to fall down. This is the same time when the first dip occurs in the inline force time series. The water column is extending in length from the back of the cylinder until the wave crest which is 1.5 diameters behind the cylinder.

At $15.2 \mathrm{~s}$, plot (c), when also the second peak occurs, the water column still distinctly exists. Even though the run-up on the back side has decreased, the free surface elevation of the outer wave flow has decreased so much that the relative height of the water column is larger than before. The water column width at the base of the water column has spread. In plot (d), at $15.3 \mathrm{~s}$, when the second dip occurs, there is a backward water flow towards the front of the cylinder. The height of the water column has drastically decreased. At $15.4 \mathrm{~s}$, plot (e), two water jets created from the initial collapse of the water column have reached the front side. Two further side waves from the collapse of the water column have started to spread towards the outer flow. In plot (f), time $15.5 \mathrm{~s}$, the remaining part of the water column is disappearing. Also, the created second pair of waves from the collapse of the water column have started to disappear. Some surface disturbance can still be seen around the cylinder.

We now turn to a more detailed investigation of the flow and forces associated with the secondary load cycle. The vertical distribution of the inline force time series is first visualized and then detailed into contributions from the front and back sides to isolate the secondary load cycle. Afterwards, we divide the pressure on the cylinder into different terms of the vertical momentum equation. Because we have all the variables in the numerical domain and around the cylinder, we can recompute the different terms. It should be mentioned that all the results presented in this section are analyses of the computations and not the measurements. However, since the validity of the model is demonstrated in the previous section, we regard the results extendable to the details of the physical flow.

To explain the flow in more detail a few terms for describing the physical events related to vertical run-up and run-down flows are given. A throw-up event is defined as shooting the water upwards in the form of a water jet. The most intuitive example of a throwup event is when the steep wave hits the cylinder (or a flat vertical wall) and shoots a water jet upwards. The pressure effect of the throw-up event is associated with positive acceleration of the water particles in the vertical direction (i.e. $\frac{\partial \rho u_{z}}{\partial t} \gg 0$ ). A suction event on the back side of the cylinder is related to the decreasing acceleration of the water column when the water below has been moved away because of the incident wave kinematics. This suction event is associated with $\frac{\partial \rho u_{z}}{\partial t} \ll 0$. A wash-down event occurs when there is a high-velocity flow on the cylinder wall and it is associated with the $\frac{u_{z} \partial \rho u_{z}}{\partial z}$ term. Finally, a catch pressure effect is observed when lower layers of the fluid decelerate the falling fluid above them and it occurs when $\frac{\partial \rho u_{z}}{\partial t}>0$ and $u_{z}<0$. Wash-down and catching usually occur simultaneously with wash-down possibly leading the process.

\subsection{Vertical distribution of inline force}

The inline force is plotted in a contour plot with the axis of time and height on the cylinder measured from the still water level ( $z$-axis of the coordinate system) in figure 8 . The plot is obtained by integration of the computational pressure on the cylinder wall in the azimuth direction. At $15.1 \mathrm{~s}$, a minimum force appears to be caused by a local 
(a)

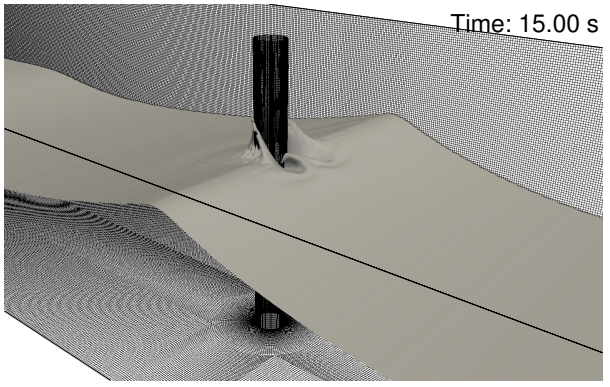

(c)

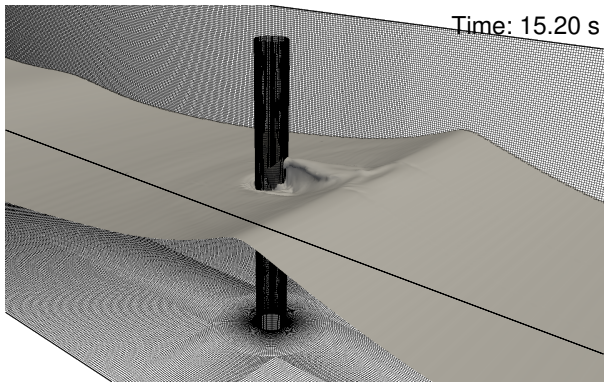

(e)

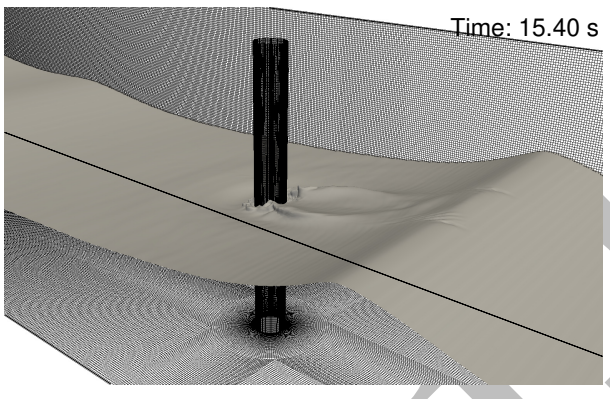

(b)

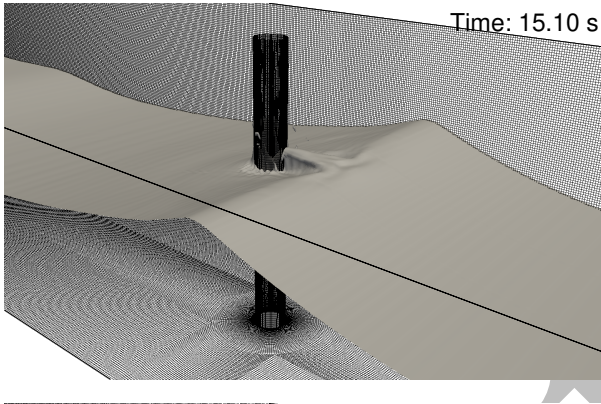

(d)
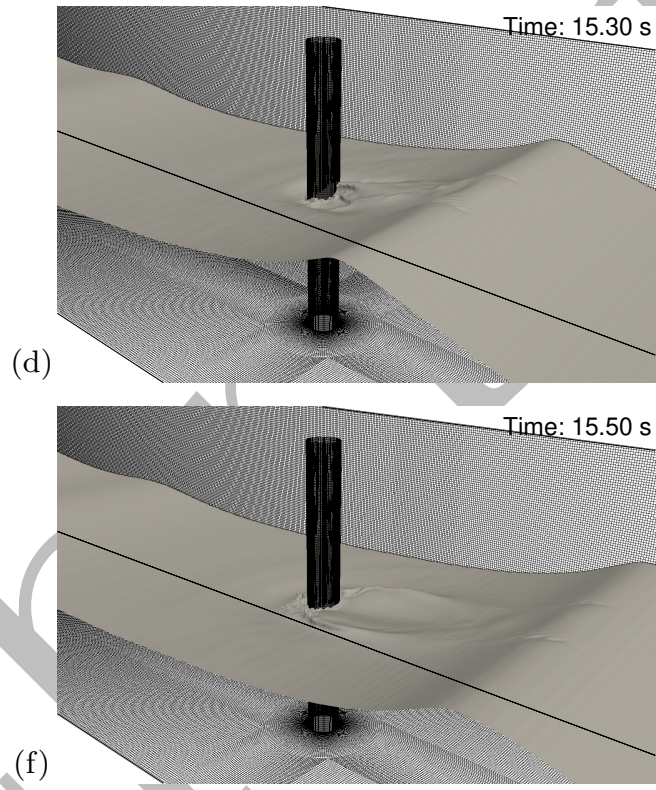

Figure 7: Snapshots of the free surface: (a) at the time of the wave crest passing the backside of the cylinder, (b) when the water column is fully formed behind the cylinder (maximum run-up behind the cylinder), (c) at the beginning of the secondary load cycle (collapse of the water column), (d) at the time of the local minimum of the secondary load cycle, (e) at the end of the secondary load cycle, (f) after the end of the secondary load cycle

low-force event close to the still water level followed by a higher force event at $15.2 \mathrm{~s}$ that propagates from above. Finally, the second force minimum at $15.32 \mathrm{~s}$ is related to another local low-force event which extends down to 1.5 times the diameter. Hence, in this plot, it is observed that the secondary load cycle is visible in the heights closer to the still water level from $15.1 \mathrm{~s}$ to $15.4 \mathrm{~s}$ and its effect extends to 1.5 diameters below the still water level.

\subsection{Azimuthal and vertical distribution of inline force}

The front side and the back side inline force per unit length of the cylinder are separately shown in figure 9 for the various vertical positions where the secondary load cycle effect was distinctly visible. The total force per unit height is shown in the blue curve while the front side force per unit height is shown in black dashed-dot lines and the back side force per unit height is shown in the black dashed line. The force-perlength from the back side is multiplied by minus one so that visual comparison with the front side is easier. In these plots, an event resembling the secondary load cycle can be 

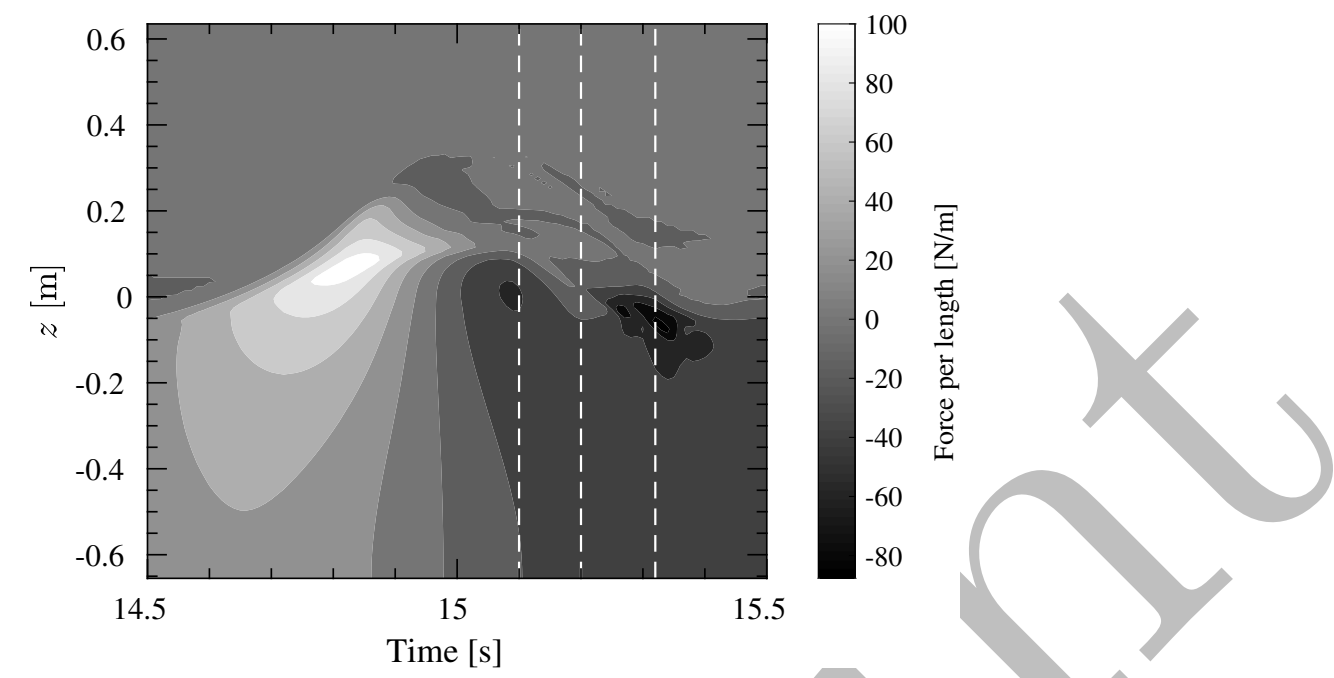

Figure 8: Contour plot of inline force per length as a function of height and time. Integrating in height leads to the total inline force time series. The white dashed lines indicate the time instants $15.1 \mathrm{~s}, 15.2 \mathrm{~s}$ and $15.32 \mathrm{~s}$, the first local minimum, the local maximum and the second local minimum of the secondary load cycle respectively.

observed in the same time period as in the total inline force time series. From $z=0.04 \mathrm{~m}$ to $z=-0.05 m$ the secondary load cycle correlates to a cycle of decrease and increase from $15.2 \mathrm{~s}$ to $15.4 \mathrm{~s}$ in the absolute value of the inline force on the back side. Similar behaviour is observed from $z=-0.09 m$ to $z=-0.21 m$ with a smaller cycle until at $z=-0.28 m$ where it has almost completely disappeared. The front side force time series shows an abrupt decrease at around $15.15 \mathrm{~s}$ to $15.30 \mathrm{~s}$ from $z=0.04 \mathrm{~m}$ to $z=-0.09 \mathrm{~m}$ at the same time as the second peak in the total force per length at the same height. Hence, the first dip and the subsequent peak in the total inline force time series cannot be related to a distinct event at either front or back. The second dip occurs when the water from the front side is washed down and a sudden stop of force decrease occurs on the back side.

\subsection{Detailed study by the vertical momentum equation}

To understand the nature of the secondary load cycle closer, the vertical momentum conservation equation is used to separate the local pressure contribution from each term. Equation 2.4 can be rewritten in cylindrical coordinates with total pressure, $p$, instead of $p^{*}$ to be used easily around the cylinder

$$
\frac{\partial \rho u_{z}}{\partial t}+u_{r} \frac{\partial \rho u_{z}}{\partial r}+\frac{u_{\theta}}{r} \frac{\partial \rho u_{z}}{\partial \theta}+u_{z} \frac{\partial \rho u_{z}}{\partial z}=-\frac{\partial p}{\partial z}+g_{z} z+\text { Viscous terms. }
$$

The surface tension term in (2.4) has been dismissed since it was not included in our computations. The vertical momentum equation has the benefit that it separates the hydrostatic terms from the other terms. The values from the OpenFOAM domain in Cartesian coordinates were transferred into the cylindrical coordinates for these analyses. In figure 10 the cylindrical coordinate system and the cells around the cylinder are shown in top view.

The polar momentum equation can be rewritten to separate the sources of pressure 
16
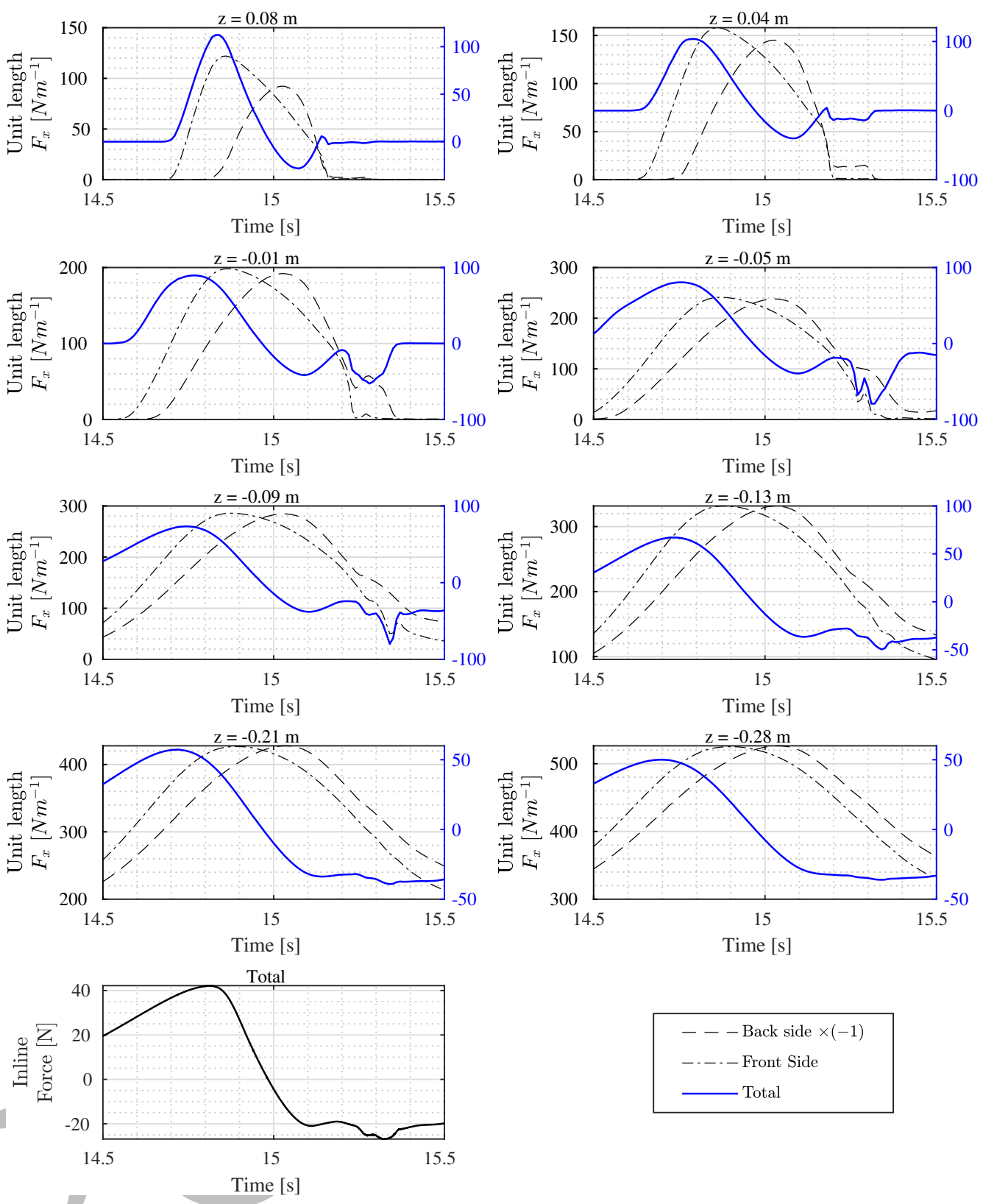

Figure 9: Separated front side, back side and total forces per unit height.

$$
\begin{aligned}
p(z)= & \int_{z}^{a t m}(\rho g) d z \\
& +\int_{z}^{a t m}(\underbrace{\frac{\partial \rho u_{z}}{\partial t}+\frac{u_{\theta}}{r} \frac{\partial \rho u_{z}}{\partial \theta}+u_{z} \frac{\partial \rho u_{z}}{\partial z}+u_{r} \frac{\partial \rho u_{z}}{\partial r}}_{\frac{\mathrm{D} \rho u_{z}}{\mathrm{D} t}}+\text { Viscous terms }) d z .
\end{aligned}
$$




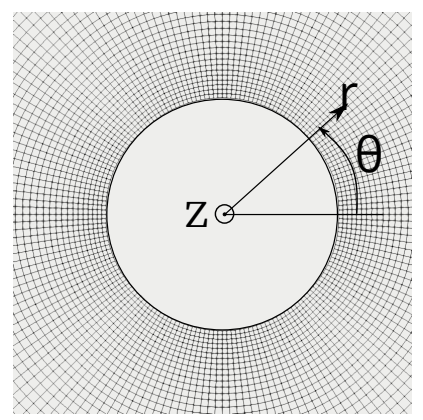

Figure 10: The cylindrical coordinates and the cells around the cylinder in top view. The cylinder is facing the waves at $\theta=\pi$.
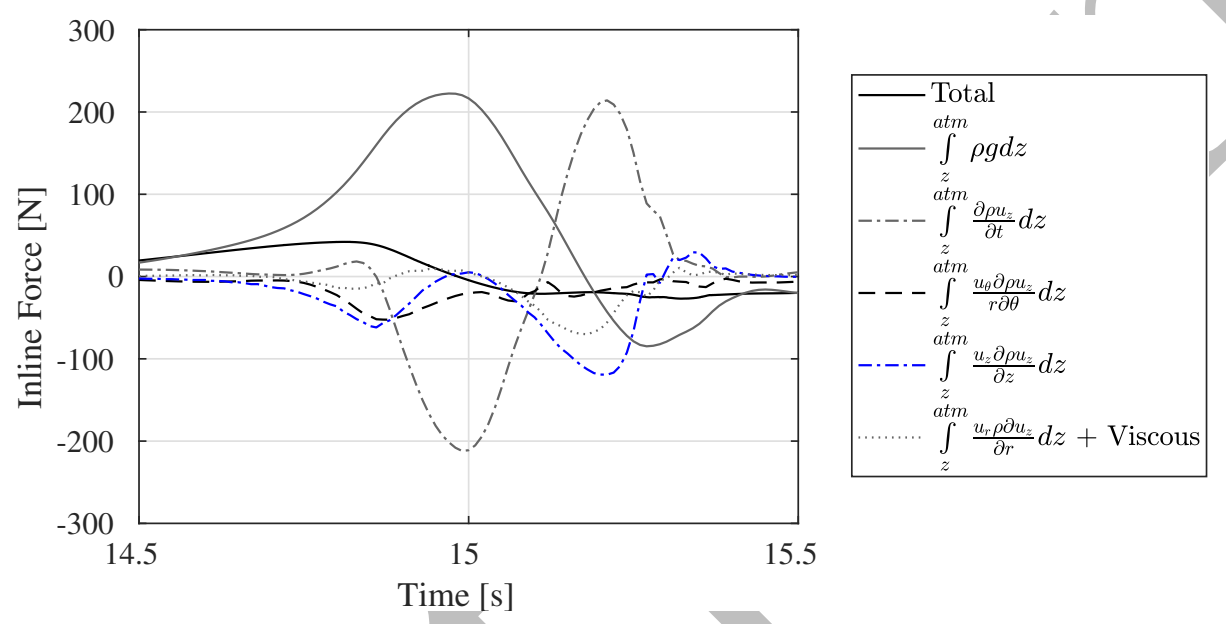

Figure 11: The forces originated from different terms of the Navier-Stokes equation in the vertical direction in cylindrical coordinate system.

To calculate the pressure contribution from the terms on the right side of (5.2) we started from the top of the domain atm (atmosphere) where the pressure was close to zero. Hence the effect of the forces from the air on the cylinder were negligible which is expected considering the ratio of the density between water and air. The total pressure on the left side is known from the computational results. The pressure contribution from $u_{r} \frac{\partial \rho u_{z}}{\partial r}$ plus the viscous terms were thus calculated from the difference between the total pressure and the other calculated terms on the right side. This process was repeated for 170 azimuth angles to get the pressure distribution around the cylinder from each term. The calculated total force from the cell centre pressure values was calculated and was found identical to the face value results. Therefore, it was justified to use the pressure and velocity component values from the cell centres closest to the cylinder wall for the analysis.

The inline force resulting from each term of (5.2) is shown in figure 11. It can be seen that the time series from the term $\frac{\partial \rho u_{z}}{\partial t}$ peaks at $15.2 \mathrm{~s}$ similar to the secondary load cycle. The hydrostatic force time series has a minimum at around $15.26 \mathrm{~s}$ which is close to the second dip of the secondary load cycle. However, since from figure 9 it is clear that the forces should be investigated more locally a side and height divided analysis of these results are next carried out.

In figures 12 and 13 the force per unit height contours plots for the major sub-terms 
of (5.2) are visualized. Each row is assigned to either the total pressure or one sub-term. From left to right, each column is attributed to the contribution from both sides, front side and back side respectively. In all the contour plots the time instants $15.10 \mathrm{~s}, 15.20 \mathrm{~s}$ and $15.32 \mathrm{~s}$ are marked for reference to the secondary load cycle with vertical dashed lines. The contours of force per meter height from the total pressure are shown in the first row of figure 12. The first plot in the first row is the same contour plot as presented in figure 8 . The middle plot visualizes the force per length on the front side of the cylinder. This plot shows some small local phenomena by increased proximity of contour lines at the same period as the secondary load cycle. At around $15.15 \mathrm{~s}$ the front side force begins to decrease faster. The change of the rate of change in the contour plot can be judged from the proximity of the contour lines. The right plot shows the forces only on the back side of the cylinder where a local cycle can be seen in the contour lines from $15.15 \mathrm{~s}$ to $15.35 \mathrm{~s}$. The local cycle can be seen in the contour lines down to 1.5 diameters from just above the still water level.

The second row includes plots of forces from the hydrostatic pressure term $\int_{z}^{a t m}(\rho g) d z$ in (5.2). In the left plot, the total hydrostatic force per unit height is shown. It is observed that due to the integrated effect of the run-up around the cylinder the hydrostatic force is positive until $15.1 \mathrm{~s}$. Afterwards, since the integrated run-up on the front side is smaller than on the back side, the inline force per unit length becomes negative. The hydrostatic force on the front side maximizes at $15.0 \mathrm{~s}$ as shown in the middle plot. Also, an increase in the hydrostatic pressure is observed after $15.32 \mathrm{~s}$. This is probably best explained by the fact that the water from the collapsed water column travels towards the front side of the cylinder because of the lower elevation of the water surface on the front side at this time. At $15.3 \mathrm{~s}$ the water from the water columns arrives at the front side so the free surface elevation increases and so the hydrostatic force increases at this point. This can also be seen in sub-plot (d) and (e) of figure 7 in which the water jets are observed to travel around the cylinder towards the front side of the cylinder. In the right plot in this row, the hydrostatic force per unit height from the back side is shown. The hydrostatic force on the back side reaches its minimum at $15.1 \mathrm{~s}$ when the run-up is maximum. This contributes to the first dip of the secondary load cycle.

In the third row, the force per length is shown, originated from the summation of the non-hydrostatic terms. An extended negative force can be observed from $15.2 \mathrm{~s}$ to $15.3 \mathrm{~s}$ close to the free surface in the middle plot which have added a tongue to the dome-shaped contours just above the still water level. In the right plot, an extended positive force can be seen around the still water level maximizing at $15.2 \mathrm{~s}$. The positive force occurs right at the second peak and is thus well in phase with the positive force peak of the secondary load cycle. Further, from inspection of figure 7, this moment is associated with a rapid decrease of water level below the water column at the back of the cylinder. Hence, to investigate the sources of the non-hydrostatic forces further, the term $\frac{\mathrm{D} \rho u_{z}}{\mathrm{D} t}$ is divided into sub-terms.

The force per length contour plots of the first three sub-terms of $\frac{\mathrm{D} \rho u_{z}}{\mathrm{D} t}$ are shown in figure 13. In the plot of the contribution of $\frac{\partial \rho u_{z}}{\partial t}$ on the front side, a positive force can be associated with the water jet created close to the free surface from the main wave reaching the front side from $14.7 \mathrm{~s}$ to $15.0 \mathrm{~s}$ (throw-up effect). Also, another region of large inline force can be seen after the run-up on the front side has started to fall from $15.05 \mathrm{~s}$ to $15.32 \mathrm{~s}$. The large inline force on the front side is closely correlated to the positive catch-pressure of the lower layers of water. On the back side, two regions of local forces can be seen. The first positive region of inline force per length is observed from around $15.10 \mathrm{~s}$ to $15.25 \mathrm{~s}$ and since it is a positive force on the back side it should be 

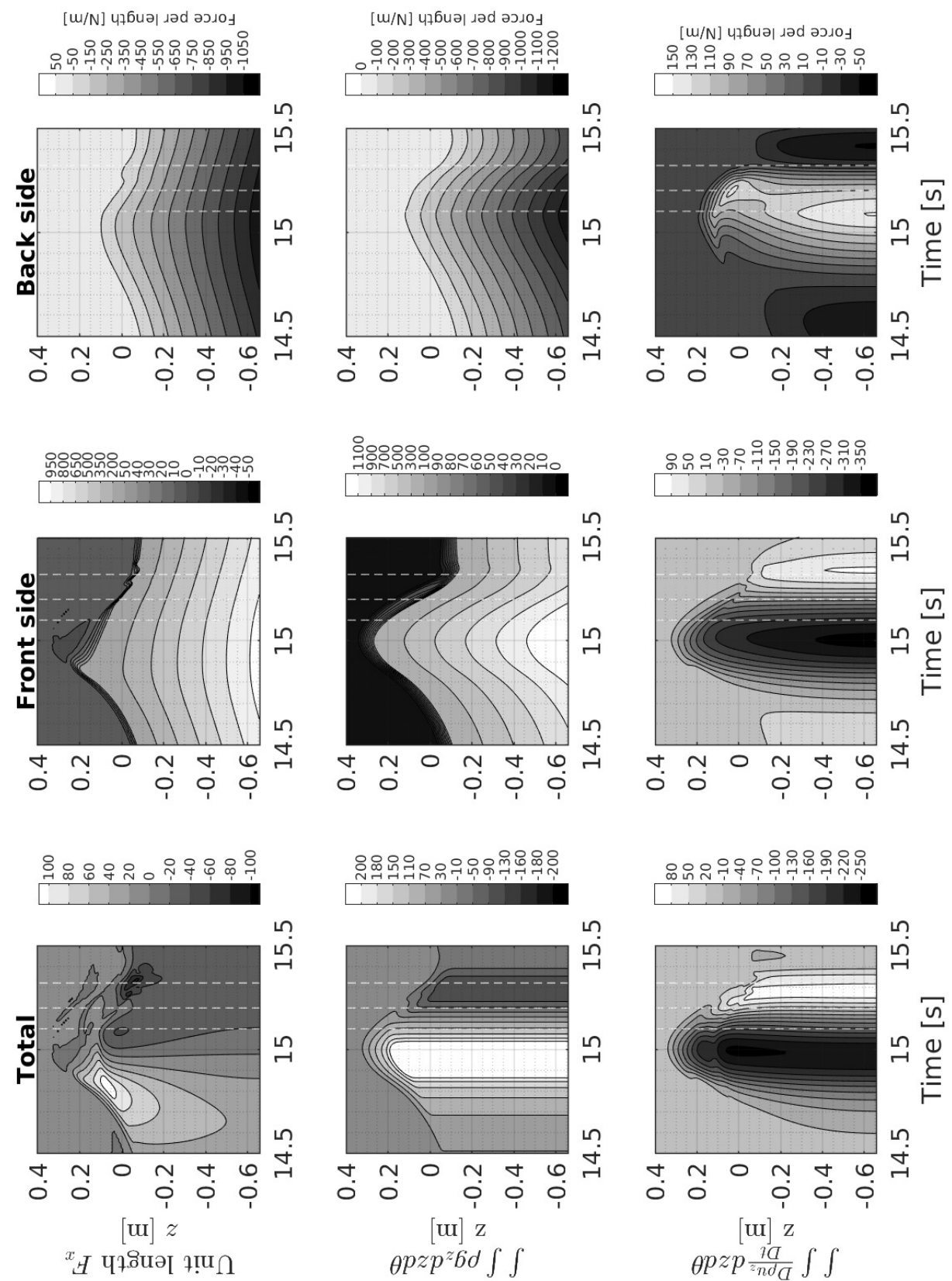

Figure 12: Force per meter separated for hydrostatic and non-hydrostatic terms in the vertical direction Navier-Stokes equation. 
caused by suction on this side. Linked to the rapid down-fall of the water level below the back-side water column, we deduce that the suction is caused by the removal of water below the water column base, because of the change of direction of the particles in the incident wave, which should be filled by the water column. The second local inline force on the back side starts from $15.2 \mathrm{~s}$ and continues until $15.35 \mathrm{~s}$ below the free surface. Since this local force is negative there should be relatively high pressure on the back side which is associated with the catch pressure of the water on the back side.

The second row of plots show the force contributions from the term $\frac{u_{\theta}}{r} \frac{\partial \rho u_{z}}{\partial \theta}$ on both sides. From these plots, the scale of this term is much smaller than the other terms and does not contribute significantly to the total local forces on the cylinder. In the third row of plots the force contributions from the term $u_{z} \frac{\partial \rho u_{z}}{\partial z}$ is presented. In the front side, the most significant contribution is from the wash-down effect which happens slightly before the catch effect. On the front side, where the water is thrown up high, the washdown effect is larger in magnitude than the catch effect. The wash-down effect starts at around $15 \mathrm{~s}$ and continues until 15.32 in two almost separate bands similar to the catch pressure effect. Its effect on the force time series can be seen in figure 9 in depths $0.04 \mathrm{~m}$ to $-0.09 \mathrm{~m}$ with the sudden decrease at around $15.2 \mathrm{~s}$. In the right bottom plot, the forces on the back side are shown where two local forces are recognizable from $15.05 \mathrm{~s}$ until 15.35 s. These two local effects are also associated with the wash-down effect of the water falling or pulled down on the back side of the cylinder. For the secondary load cycle, although the wash-down, catch and hydrostatic pressures on both sides play roles, the most important contribution is from the suction on the back side of the cylinder.

Snapshots of the total, hydrostatic and non-hydrostatic pressures on the cylinder are plotted in figure 14 for the time instants of $15.20 \mathrm{~s}$ and $15.25 \mathrm{~s}$. In the middle plot in the first row, the suction region can be distinctly observed in the base of the water column on the back side of the cylinder. The suction effect is maximum at around $15.2 \mathrm{~s}$ according to right bottom plot of figure 12 , right top plot of figure 13 and the non-hydrostatic pressure contour plots. In figure 14, low pressure bands can be observed on the front side of the cylinder around the free surface which correlates to the wash-down effect of the water on this side. From the middle plot in the second row, and other snapshots not presented here for brevity, the wash-down effect on the front side continues its effect as a negative force until 15.32. The hydrostatic pressure contour plots show that from $15.2 \mathrm{~s}$ to $15.25 \mathrm{~s}$ the hydrostatic pressure has decreased more on the front side than on the back side which is consistent with the results shown in the integrated force time series of the hydrostatic forces in figure 11. In summary, the secondary load cycle is associated with non-hydrostatic effects and is mainly caused by suction at the back side of the cylinder. This is in line with the suggestion of Grue et al. (1994). The detailed analysis further shows that the suction arises when the water level decreases rapidly while the water column has only just begun its downward acceleration after its maximum run-up.

\section{The effect of no-slip boundary condition on the cylinder wall}

While the present results with the inclusion of turbulence and the wall boundary layer provide good reproduction of the secondary load cycle, we note that previous works with slip condition and no turbulence modelling have shown similarly good reproductions. Paulsen et al. (2014b) presented such results where they observed vortices at the backside of the cylinder and linked them to the creation of the secondary load cycle. It is thus relevant to investigate the role of the boundary layer for the creation of the secondary load cycle and to discuss the origin of the vortices in the slip cases.

To investigate these matters, additional computations with slip boundary condition 

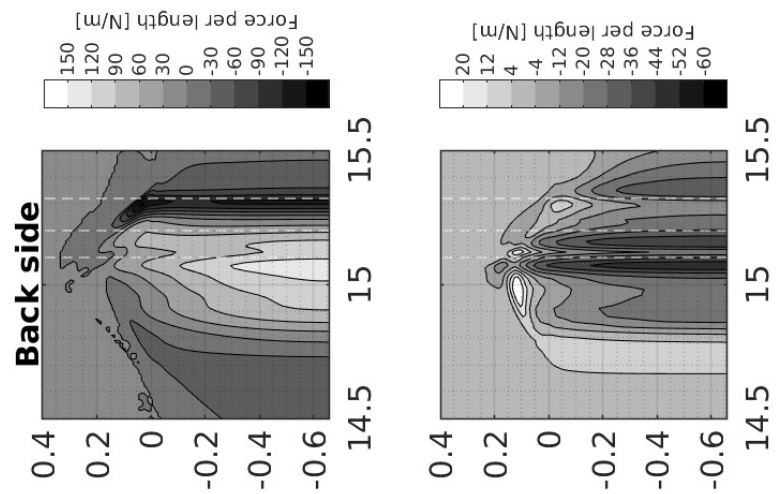

[u/N] чұбธә גәd әวגо

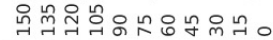

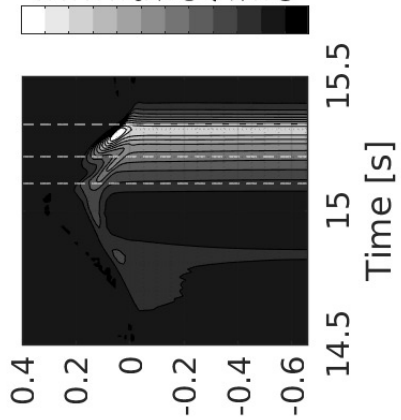

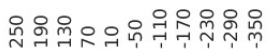

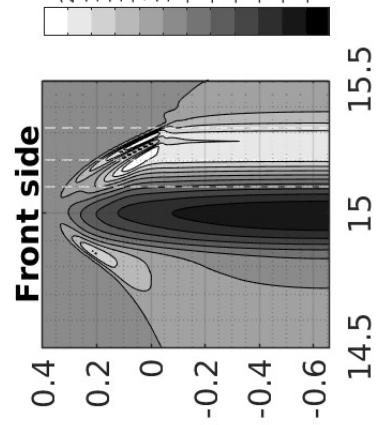

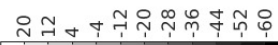

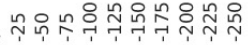
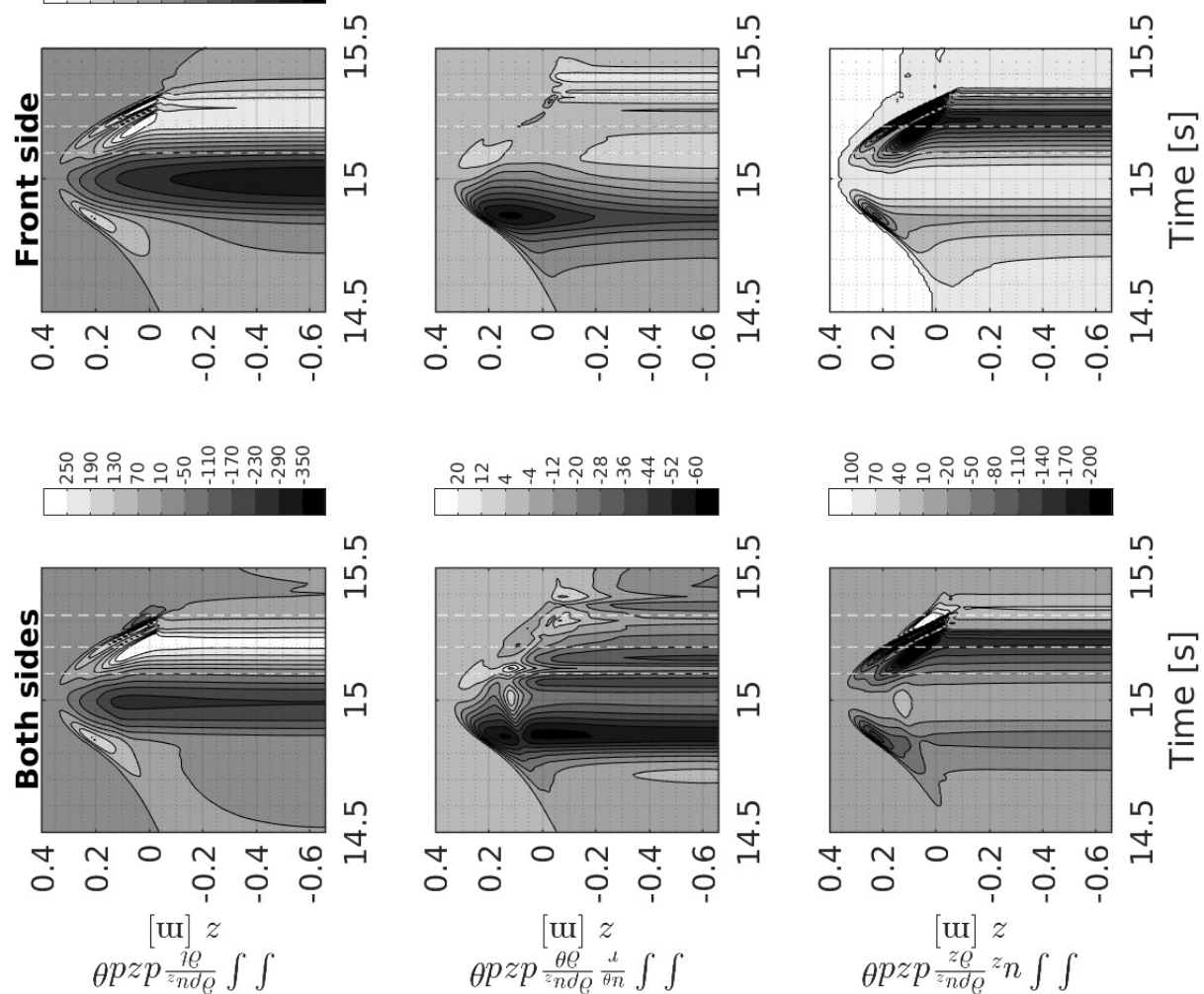

Figure 13: Force per meter $\frac{\partial \rho u_{z}}{\partial t}, \frac{u_{\theta}}{r} \frac{\partial \rho u_{z}}{\partial \theta}$ and $u_{z} \frac{\partial \rho u_{z}}{\partial z}$ terms. 

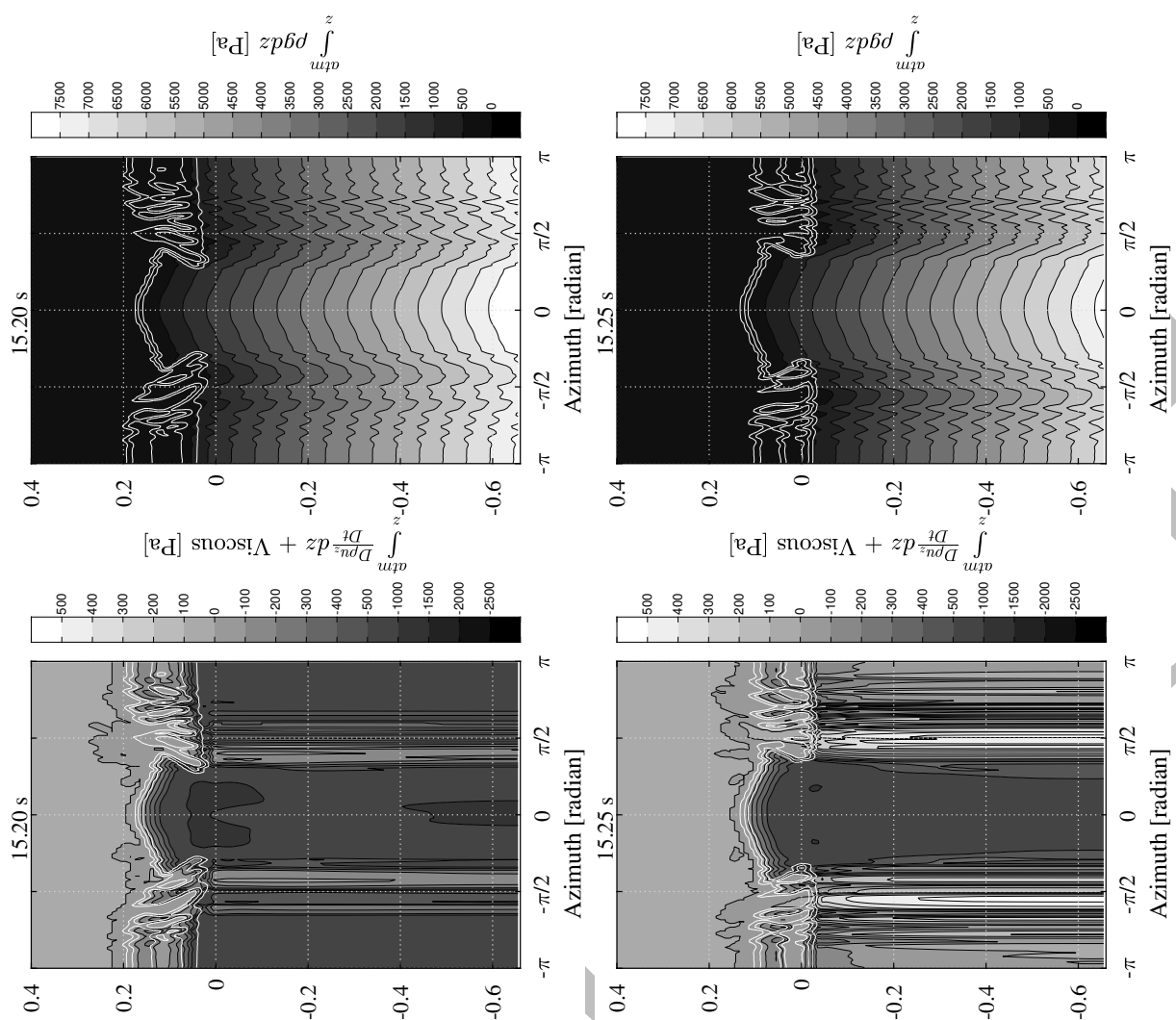

$\left[\mathrm{e}_{\mathrm{d}}\right]{ }^{10707} \mathrm{~d}$
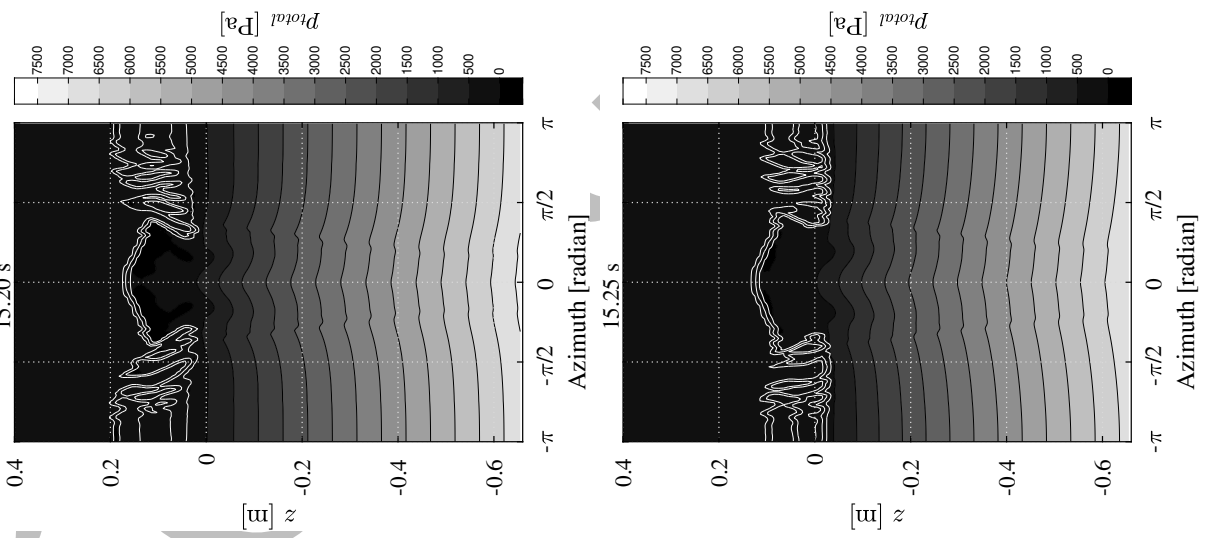

Figure 14: Pressure contours from total, non-hydrostatic and hydrostatic sources at times $15.20 \mathrm{~s}$ and $15.25 \mathrm{~s}$.

on the cylinder wall and neglecting the turbulent viscosity, $\nu_{t}$, were made. In both sets of results, a kinematic fluid viscosity of $\nu_{\text {fluid }}=1.010^{-6} \mathrm{~m}^{2} / \mathrm{s}$ was used which would allow dissipation of the flow and the vorticity similar to the real case. In figure 15, the measured inline force time series is plotted for the slip and no-slip results along with their difference. The time series from the no-slip solution is slightly closer to the measurements and from the peak of the force and throughout the secondary load cycle, the force for the slip condition is consistently smaller than for the no-slip condition. Nevertheless, the 


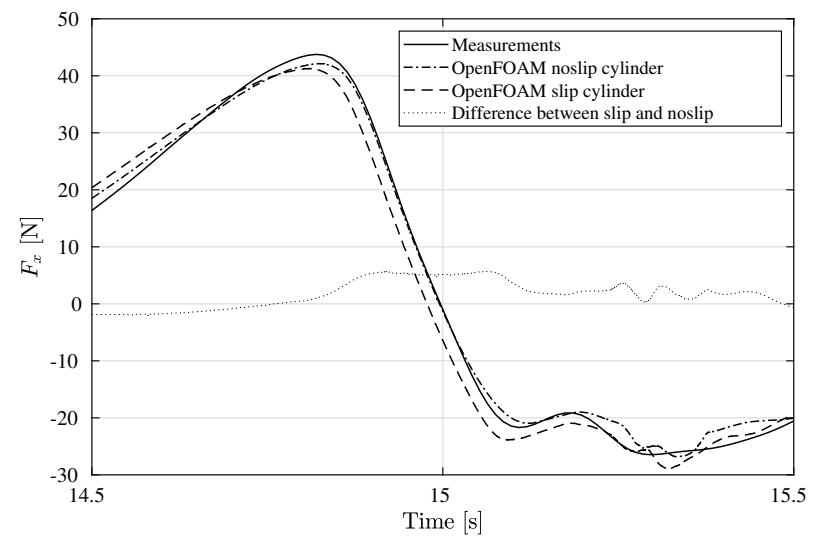

Figure 15: Comparison of the inline force in the computations with no-slip boundary condition and with slip boundary condition on the cylinder.

secondary load cycles in the two solutions show remarkable agreement with approximately identical durations and magnitudes. These results suggest that the secondary load cycle is only slightly affected by the cylinder boundary layer.

The investigation of the formation of vortices is shown in figure 16. In this figure, contour plots of the vorticity just after the outer flow reversal around the cylinder are shown at $\mathrm{z}=-0.10 \mathrm{~m}$. The left column shows the results from the slip case and the right column shows the results of the no-slip case. For the slip results, vorticity is formed from about $14.75 \mathrm{~s}$, in the vicinity of the cylinder wall, mainly from $\theta=90^{\circ}$ to the back side of the cylinder. The vorticity is advected to the backside of the cylinder where a vortex pair is formed at $\mathrm{t}=14.95 \mathrm{~s}$. This process is remarkably similar to that of the no-slip results. Here the vorticity is produced along the full circumference of the cylinder from about $14.65 \mathrm{~s}$ when the outer flow direction becomes positive. The vorticity is advected to the backside of the cylinder and a vortex pair emerges at $t=14.95 \mathrm{~s}$. From these plots it seems that even though, the process of the vortex generation does not largely depend on the boundary condition on the cylinder in these cases, the magnitude and the extent of the vortices depend on it and are larger in the no-slip case.

We note here, though, that although the slip condition may be associated with the potential flow, the full computational flow is not inviscid. Our computations for the slip boundary condition cases are solutions of the Navier-Stokes equations with an effective kinematic viscosity from the fluid and further contribution from truncation errors in the numerical schemes $\left(\nu_{\text {effective }}=\nu_{\text {fluid }}+\nu_{\text {numerical }}\right)$.

To examine the influence of the vortices downstream of the cylinder on the creation of the secondary load cycle, a bulk vorticity measure was defined as the integrated vorticity in a plane. This method helps to visualize the time scales of the vortices relative to that of the secondary load cycle. We chose a vertical plane at $z=-0.15 \mathrm{~m}$, which is well below the lowest position of the free surface, and still within the vertical extent of the secondary load cycle (see figure 8). The bulk vorticity measure is defined as

$$
\Omega=\int_{A}|\vec{\omega}| d A
$$

where $A$ denotes the area from $x=7.1 \mathrm{~m}$ and $y=1.9 \mathrm{~m}$ to $x=7.5 \mathrm{~m}$ and $y=2.0 \mathrm{~m}$. The cylinder centre is located at $x=7.3 \mathrm{~m}$ and $y=2.0 \mathrm{~m}$. Also, a closer study by mesh refinement was performed to investigate the effect of the mesh on the magnitude of vorticity close to the cylinder. 


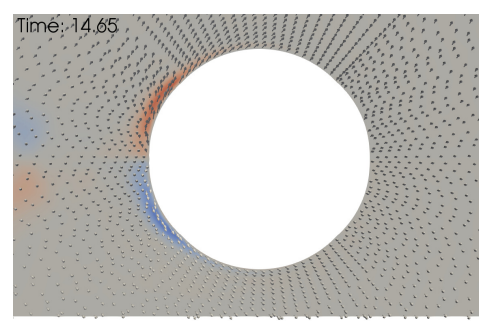

(a)
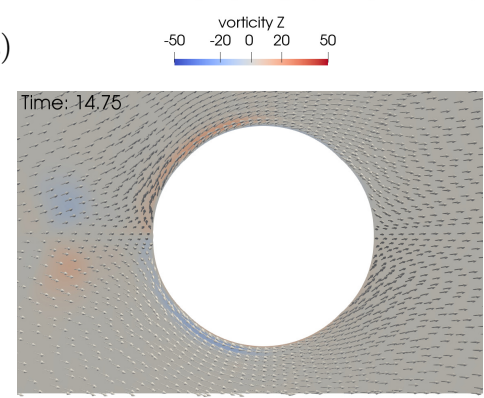

(c)
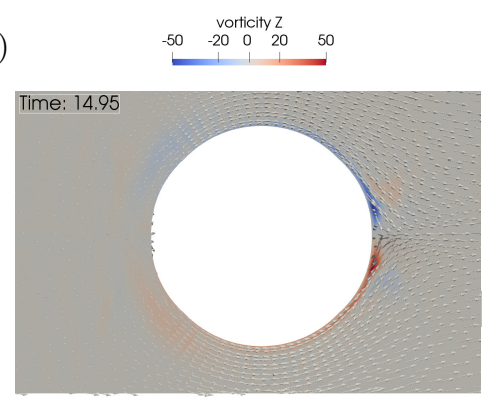

(e)
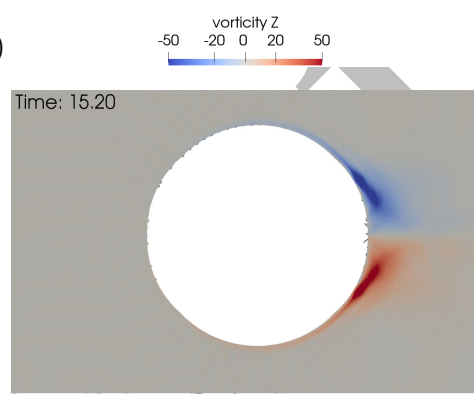

(g)

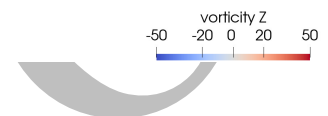

(b)

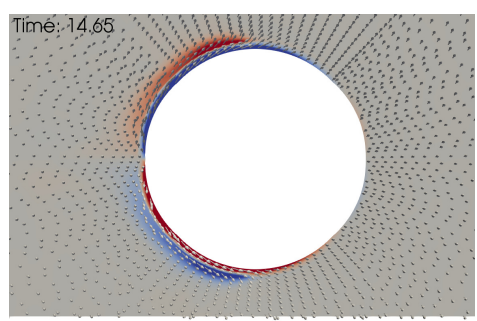

vorticity $Z$

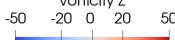

(d)

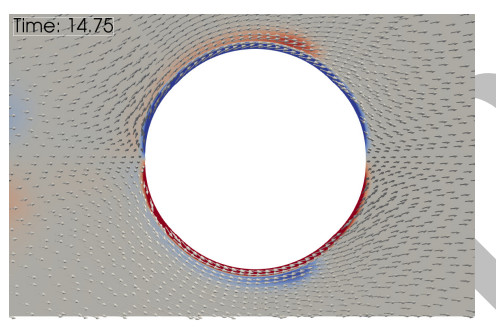

vorticity Z

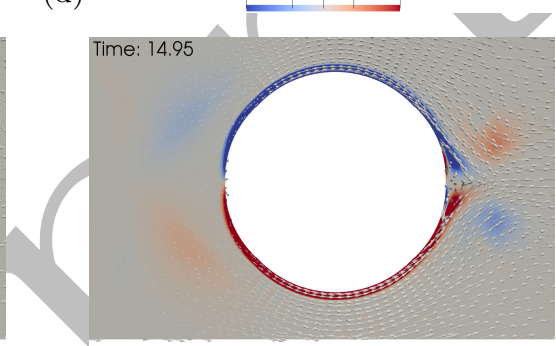

(f)

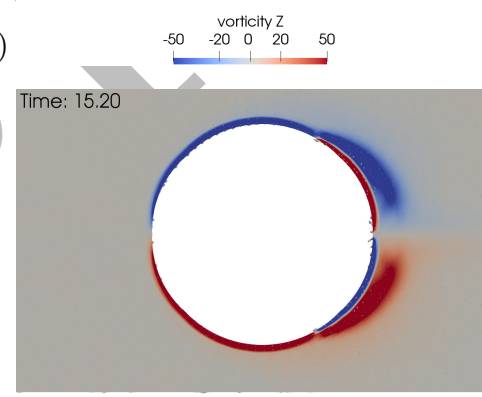

(h)

Figure 16: Appearance of vortices around the cylinder with passage of steep waves in diffraction zone at $z=-0.10 \mathrm{~m}$. Vorticity is shown with filled contours. Left column: slip. Right column: no-slip.

In figure $17, \Omega$ is shown for mesh resolutions of 3,8 and 24 million cells for both slip and no-slip conditions. The overall magnitude of the vorticity is larger in the noslip results due to the contribution from the wall boundary layer. Both solutions show a general increase in vorticity as expected. However, the time scale of the changes in both slip and no-slip results are larger than the time scale of the secondary load cycle. This observation indicates that the vortices cannot cause the secondary load cycle. In addition, from figure 17 , the mesh resolution is seen to have only a minor effect on 

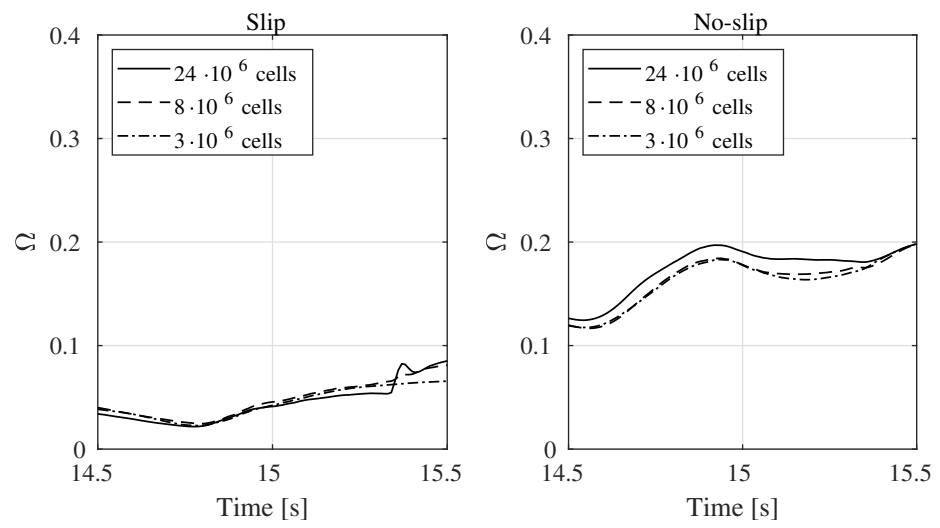

Figure 17: Integrated magnitude of the vorticity in a plane at $z=-0.15 \mathrm{~m}$ below the still water level close to the cylinder for different mesh resolutions and boundary conditions (slip vs no-slip) on the cylinder wall.
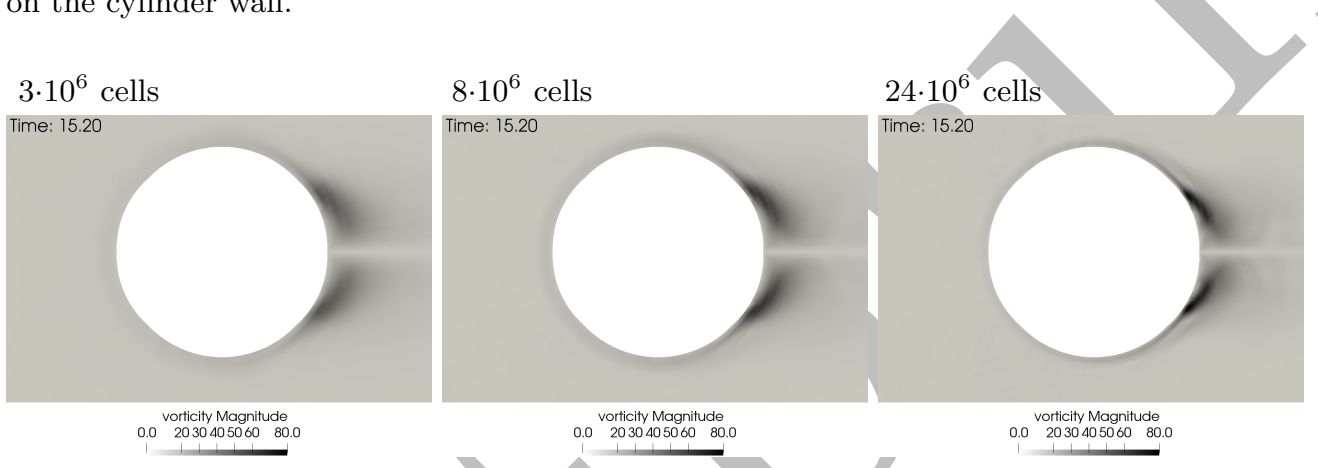

Figure 18: Snapshots of the vorticity magnitude in a plane at $z=-0.05 \mathrm{~m}$ for slip boundary condition with three different mesh resolutions.

the results, which might mean that the vortices at the rear side of the cylinder are not created from the numerical errors that are mesh dependent. For the no-slip condition, the vorticity level increases with resolution, which is consistent with a reduced magnitude of the numerical viscosity and thus reduced dissipation. The same trend is not seen for the slip results. The difference in the no-slip results at $15.35 \mathrm{~s}$ for the finest grid is caused by the proximity of the free surface at this time.

Further, in figure 18, snapshots of the vorticity magnitude in a plane at $z=-0.05 \mathrm{~m}$ for slip computations with the three mesh resolutions are shown. These plots show that the magnitude increases to a larger maximum level and the eddies are more concentrated for the finer meshes. This is consistent with an increased numerical dissipation for the coarser grids. The inline force time series for different mesh resolutions for slip and no-slip computations are further shown in figure 19. In the slip cases, there are small differences around the peak of the time series. In the no-slip cases, the mesh-induced differences are smaller around peak time. In both cases, the secondary load cycle is broadly similar across the grids. This is further detailed in table 1, where the normalized secondary load cycle amplitude (range of the secondary load cycle divided by the range of the total inline force time series) is presented. The normalized amplitudes are identical or very close between the different mesh resolutions. However, the normalized amplitude is larger for the no-slip cases.

From these plots, and detailed investigation of the computations, one can conclude 

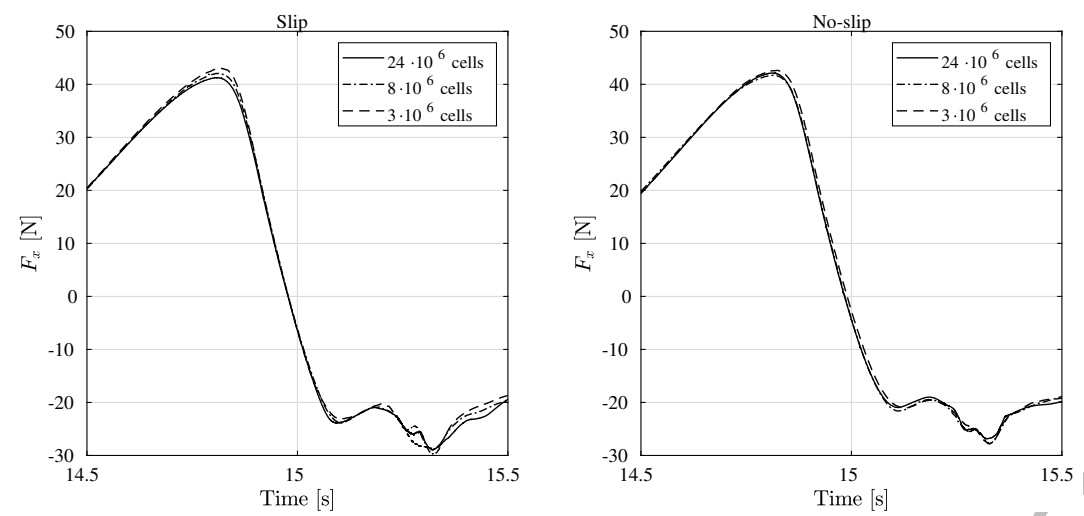

Figure 19: Inline force time series for different mesh resolutions for slip and no-slip computations.

Number of cells slip no-slip

$\begin{array}{lll}24 \cdot 10^{6} & 0.10 & 0.11 \\ 8 \cdot 10^{6} & 0.10 & 0.12 \\ 3 \cdot 10^{6} & 0.10 & 0.12\end{array}$

Table 1: The amplitude of secondary load cycle normalized by the total amplitude of the inline force.

that the vortices do not contribute largely to the secondary load cycle creation or to its amplitude and that the vortices downstream of the cylinder are not strongly influenced by the mesh resolution. However, the reason for the creation of the vortices behind the cylinder is still unknown. In general, such vortices are created because of flow separation. In the cases with slip boundary conditions on the cylinder, one expects to have no separation. Detailed investigation were conducted to investigate if presence of sharp corners in the meshes could explain the observed production of vorticity along the cylinder wall. Although no such mesh created sharp corners were visible, this remains a plausible source for the observed initial vorticity. Overall, we observed that the vorticity generation and location of the separation point are to a large extent mesh-independent. This observation may suggest that the separation is governed by the outer flow. For inviscid flow around the cylinder, where no separation occurs, the fluid travels against an adverse pressure gradient from $\theta=\pi / 2$. In the present flow condition, the outer pressure gradient of the wave motion acts to decelerate the flow further at the time of separation. This can thus help to cause separation. Still, viscosity is needed to enable separation which probably comes partly from the numerical errors, and partly from the fluid viscosity and can contribute to further vorticity production in the outer flow after the initial flow separation. The grid-independence of the separation point may be linked to the classical concept of flow regimes, defined by the outer flow and the magnitude of viscosity. Similar to the weak dependence of separation point to Reynolds number for classical subcritical flow past a cylinder, a change in numerical viscosity through an altered mesh resolution was not found to influence the flow regime strongly in the present case. 


\section{Summary and discussion}

The secondary load cycle for a focused wave group passing a vertical circular cylinder has been investigated experimentally and numerically. The experiment consisted of eight repeats of a focused wave group in the parameter range typical for offshore wind turbine storm conditions. The wave chosen was a 3-hour New Wave for a 10 year return period climate in typical North Sea conditions close to the German Bight. A two-phase numerical model was set up to reproduce the experiment. The model was validated with a satisfactory agreement for cases of turbulent flow over a wall, oscillatory boundary layer, steady flow around a cylinder and oscillatory flow around a cylinder. The latter flow was validated for $R e=\left\{5.8 \cdot 10^{4}, 9 \cdot 10^{4}, 1.7 \cdot 10^{5}\right\}$ and $K C=\{6,12,18\}$ which is representative for the experimental flow conditions of the focused wave group.

Afterwards, a detailed study of the secondary load cycle was performed using the validated model. By comparison of the run-up and run-down time series at the front and back of the cylinder, it was found that the time scales of these flows are too long to explain the load cycle through hydrostatic effects. Next, by inspection of the inline force distribution, we found that the secondary load cycle is associated with local phenomena affecting the cylinder in a region from just above the still water level and 1.5 diameters down. Further division into the contributions from the front and back side reveals that the secondary load cycle is correlated with short period cycles in the time series from both the front side and back side.

For further investigation, the forces from hydrostatic and non-hydrostatic terms of the vertical momentum equation were calculated and it was observed that the secondary load cycle is mostly correlated with forces from the non-hydrostatic term. More precisely, the main cause of the secondary load cycle is a suction pressure which occurs just after the maximum run-up of the water column at the back of the cylinder. At this time the free surface elevation of the outer wave flow falls rapidly. The needed downward acceleration of the water column generates the suction pressure at the root of the column which constitutes the local peak of the secondary load cycle. The first dip, preceding this peak, is enhanced by the hydrostatic pressure of the water column during its maximum height, while the second dip is enhanced by wash-down effects on the front side and the total hydrostatic pressure from both sides.

Further observations during the flow include

- A throw-up pressure at $14.85 \mathrm{~s}$ on the front side which contributes to the peak of the total inline force at the same time.

- Free-fall conditions for the run-up at the front side. At $15.0 \mathrm{~s}$ this run-up is maximum. However, since the water is thrown up freely, the hydrostatic pressure is cancelled out by the acceleration term $\frac{\partial \rho u_{z}}{\partial t}$.

- Fall down at $15.2 \mathrm{~s}$ on the front side with faster velocity than free fall. This leads to a low-pressure region, created just below the free surface.

- A similar wash-down pressure at $15.32 \mathrm{~s}$ on the back side is observed.

In the last section, emphasis was given to the effect of the wall boundary layer on the vortex formation behind the cylinder and the secondary load cycle. While vortices occur for computations with both slip and no-slip boundary conditions, we found that the time seale of vortex formation is too slow to explain the secondary load cycle. Further, although differences between the two results could be observed, the secondary load cycle appears to be similar for both slip and no-slip results. The occurrence of flow separation for slip case was investigated and attributed to grid effects and numerical viscosity in combination with the pressure gradient of the outer flow. No strong mesh-dependence was found. 
An aspect not covered in the study is possible scale-effects relative to full scale. At the small scale in the lab, surface tension may act to damp the local run-up and to keep the mound flow more coherent than in full scale. Also the presence of small air bubbles from previous breaking and run-up or wash-down can make the free surface less uniform at larger scale. The suction effect, however, is caused by the total flow which will scale according to the Froude law. We thus expect that the main flow is largely scaleable and that the qualitative conclusions of the investigation will apply also at full scale.

The study demonstrates the strength of combined experimental-numerical investigations, where further and even very local details can be obtained from the model after careful reproduction of a physical test. While the secondary load cycle has been known for more than two decades and has often been associated with the flow at the cylinder back, the present investigation provides an accurate description of its cause.

\section{Acknowledgement}

This work was funded by the Innovation Fund Denmark and other partners as part of DeRisk project with grant number 4106-00038B. This support is gratefully acknowledged by the authors. Martin Dixen, DHI Denmark, is thanked for access to the experimental data. Bjarke Eltard, DTU Mechanical Engineering, is thanked for discussions on the OpenFOAM computations.

\section{Declaration of Interests}

The authors report no conflict of interest.

\section{REFERENCES}

Bredmose, H., Dixen, M., Ghadirian, A., Larsen, T., Schløer, S., Andersen, S., Wang, S., Bingham, H., Lindberg, O., Christensen, E., Vested, M., Carstensen, S., Engsig-Karup, A., Petersen, O., Hansen, H., Mariegaard, J., Taylor, P., Adcock, T., Obhrai, C., Gudmestad, O., Tarp-Johansen, N., Meyer, C., Krokstad, J., Suja-Thauvin, L. \& Hanson, T. 2016 DeRisk Accurate Prediction of ULS Wave Loads. Outlook and First Results. Energy Procedia 94, 379-387.

Bredmose, H., Hunt-Raby, A., Jayaratne, R. \& Bullock, G. N. 2010 The ideal flip-through impact: Experimental and numerical investigation. Journal of Engineering Mathematics 67 (1), 115-136.

Brown, S. A., Magar, V., Greaves, D. M. \& Conley, D. C. 2014 An Evaluation of RANS Turbulence Closure Models for Spilling Breakers. Coastal Engineering Proceedings 1 (34), 5.

Chaplin, J. R., Rainey, R. C. T. \& YEMm, R. W. 1997 Ringing of a vertical cylinder in waves. Journal of Fluid Mechanics 350, 119-147.

Dean, R. \& Dalrymple, R. A. 1991 Water wave mechanics for engineers and scientists. World Scientific Publishing Company.

Deshrande, S. S., Anumolu, L. \& Trujillo, M. F. 2012 Evaluating the performance of the two-phase flow solver interFoam. Computational Science \& Discovery 5 (1), 014016.

Devolder, B., Rauwoens, P. \& Troch, P. 2017 Application of a buoyancy-modified k- $\omega$ SST turbulence model to simulate wave run-up around a monopile subjected to regular waves using OpenFOAM@. Coastal Engineering 125 (June 2016), 81-94.

Engsig-Karup, A. P., Bingham, H. B. \& Lindberg, O. 2009 An efficient flexible-order model for 3D nonlinear water waves. Journal of Computational Physics 228 (6), 2100-2118.

Fredsøe, J., Sumer, B. M., Kozakiewicz, A., Chua, L. H. \& Deigaard, R. 2003 Effect of externally generated turbulence on wave boundary layer. Coastal Engineering 49 (3), $155-183$. 
Ghadirian, A., Bredmose, H. \& Dixen, M. 2016 Breaking phase focused wave group loads on offshore wind turbine monopiles. Journal of Physics: Conference Series 753 (9), 092004.

Ghadirian, A., Bredmose, H. \& Schløer, S. 2017 Prediction of the shape of inline wave force and free surface elevation using First Order Reliability Method (FORM). Energy Procedia 137 (January), 162-176.

GodA, Y. \& Suzuki, T. 1976 Estimation of Incident and Reflected Waves in Random Wave Experiments. In proceedings of the 15th Conference on Coastal Engineering pp. 828-845.

Greenshields, C. J. 2015 OpenFOAM-3.0.1 (December).

GRUE, J. 2002 On four highly nonlinear phenomena in wave theory and marine hydrodynamics. Applied Ocean Research 24 (5), 261-274.

Grue, J., BJørshol, G. \& Strand, Ø. 1994 Nonlinear wave loads which may generate "Ringing" responses of offshore structure. In Ninth International Workshop on Water Waves and Floating Bodies.

Grue, J. \& Huseby, M. 2002 Higher harmonic wave forces and ringing of vertical cylinders. Applied Ocean Research 24 (4), 203-214.

Hirt, C. W. \& Nichols, B. D. 1981 Volume of fluid (VOF) method for the dynamics of free boundaries. Journal of Computational Physics 39 (1), 201-225.

Jacobsen, N. G. 2011 A Full Hydro- and Morphodynamic Description of Breaker Bar Development. PhD thesis.

Jacobsen, N. G. 2017 waves2Foam Manual. Tech. Rep. August. Deltares.

Jacobsen, N. G., Fuhrman, D. R. \& Fredsøe, J. 2012 A wave generation toolbox for the opensource CFD library: OpenFoam. International Journal for Numerical Methods in Fluids 70 (9), 1073-1088.

Jensen, B. L., Sumer, B. M. \& Fredsoe, J. 1989 Turbulent oscillatory boundary layers at high Reynolds numbers. Journal of Fluid Mechanics 206, 265-297.

Jose, J., Choi, S.-J., Giljarhus, K. E. T. \& Gudmestad, O. T. 2017 A comparison of numerical simulations of breaking wave forces on a monopile structure using two different numerical models based on finite difference and finite volume methods. Ocean Engineering 137 (1), 78-88.

von Karman, T. 1931 Mechanical Similitude and Turbulence. National Advisory Committee for Aeronautics - Technical Memorandum .

Kristiansen, T. \& Faltinsen, O. M. 2017 Higher harmonic wave loads on a vertical cylinder in finite water depth. Journal of Fluid Mechanics 833, 773-805.

Larsen, B. E., Fuhrman, D. R. \& Roenby, J. 2018 Performance of interFoam on the simulation of progressive waves. arXiv preprint, arXiv: 1804.01158.

Menter, F., Kuntz, M. \& Langtry, R. 2003 Ten years of industrial experience with the SST turbulence model. Turbulence, heat and mass transfer 4 (1), 625-632.

Paulsen, B. T. 2013 Efficient computations of wave loads on off shore structures. PhD thesis.

Paulsen, B. T. Bredmose, H. \& Bingham, H. B. $2014 a$ An efficient domain decomposition strategy for wave loads on surface piercing circular cylinders. Coastal Engineering 86, $57-76$.

Paulsen, B. T., Bredmose, H., Bingham, H. B. \& Jacobsen, N. G. $2014 b$ Forcing of a bottom-mounted circular cylinder by steep regular water waves at finite depth. Journal of Fluid Mechanics 755, 1-34.

RAINEy, R. C. T. 2007 Weak or strong nonlinearity: The vital issue. Journal of Engineering Mathematics 58 (1-4), 229-249.

Riise, B. H., Grue, J., Jensen, A. \& Johannessen, T. B. 2018 A note on the secondary load cycle for a monopile in irregular deep water waves. Journal of Fluid Mechanics 849, R1.

Rosetti, G. F., Vaz, G. \& Fujarra, A. L. C. 2012 URAnS Calculations for Smooth Circular Cylinder Flow in a Wide Range of Reynolds Numbers: Solution Verification and Validation. Journal of Fluids Engineering 134 (12), 121103.

Stringer, R. M., ZANG, J. \& Hillis, A. J. 2014 Unsteady RANS computations of flow around a circular cylinder for a wide range of Reynolds numbers. Ocean Engineering 87, 1-9.

Sumer, B. M. \& Fredsøe, J. 2006 Hydrodynamics Around Cylindrical structures. World Scientific Publishing Company.

Tromans, P. S., Anatruk, A. R. \& Hagemeijer, P. 1991 A New Model for the Kinematics 
<smiles>C[C@H]1C=C[C@@H]1[Te]</smiles> 


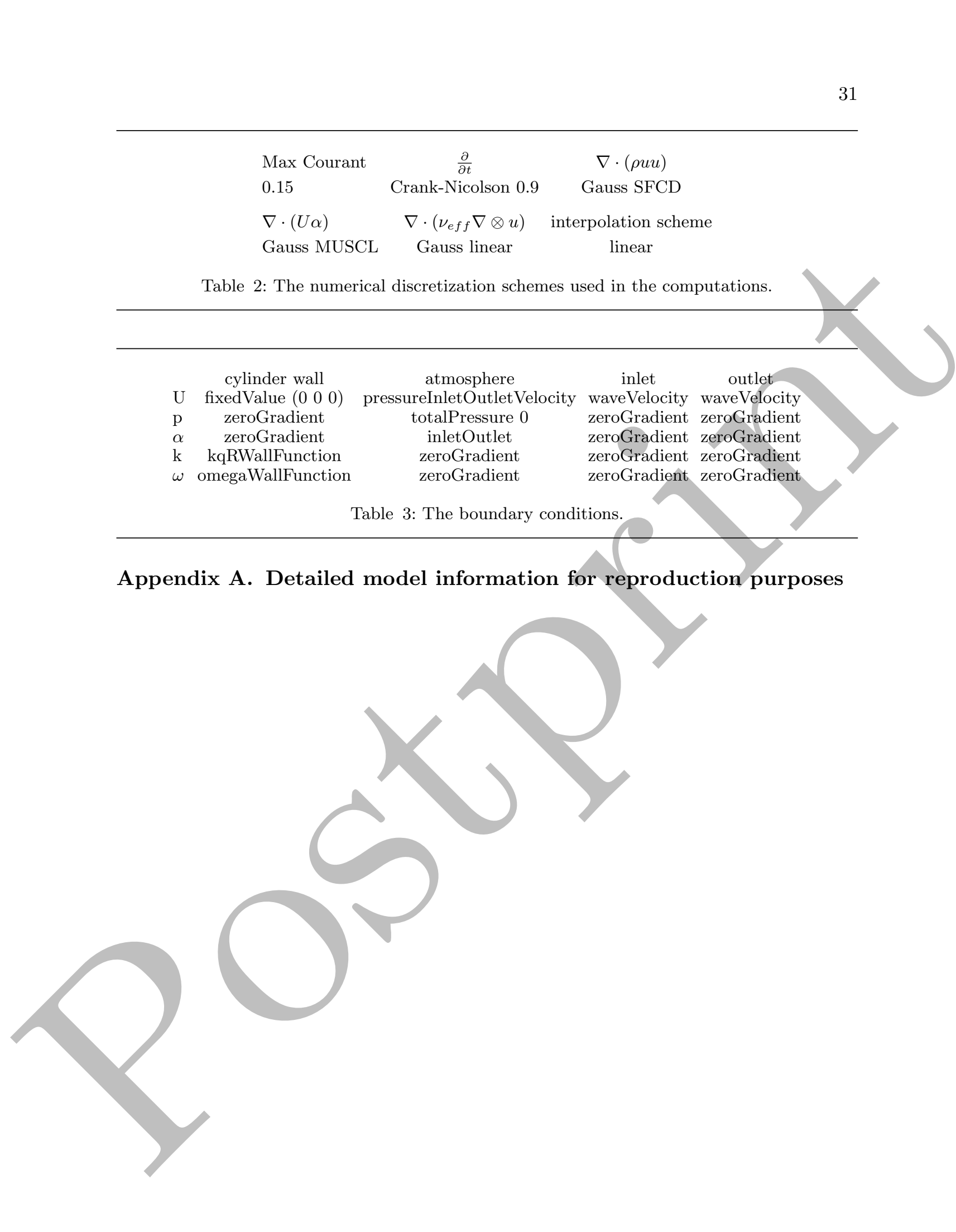

